\title{
Some relational structures with polynomial growth and their associated algebras I: Quasi-polynomiality of the profile
}

\author{
Maurice Pouzet* \\ Université Claude-Bernard, Lyon1, ICJ, \\ 43, Bd. du 11 Novembre 1918, Villeurbanne, F-69622 \\ Department of Mathematics and Statistics, The University of Calgary, \\ Calgary, Alberta, Canada \\ pouzet@univ-lyon1.fr \\ Nicolas M. Thiéry \\ Université Paris-Sud, Laboratoire de Mathématiques d'Orsay, Orsay, F-91405; \\ CNRS, Orsay, F-91405 \\ nthiery@users.sf .net \\ Dedicated to Adriano Garsia, with warmth and admiration
}

Submitted: Mar 30, 2012; Accepted: Mar 27, 2013; Published: Apr 9, 2013

Mathematics Subject Classification: 05E99

\begin{abstract}
The profile of a relational structure $R$ is the function $\varphi_{R}$ which counts for every integer $n$ the number $\varphi_{R}(n)$, possibly infinite, of substructures of $R$ induced on the $n$-element subsets, isomorphic substructures being identified. If $\varphi_{R}$ takes only finite values, this is the Hilbert function of a graded algebra associated with $R$, the age algebra $\mathbb{K} \mathcal{A}(R)$, introduced by P. J. Cameron.

In this paper we give a closer look at this association, particularly when the relational structure $R$ admits a finite monomorphic decomposition. This setting still encompass well-studied graded commutative algebras like invariant rings of finite permutation groups, or the rings of quasi-symmetric polynomials. We prove that $\varphi_{R}$ is eventually a quasi-polynomial, this supporting the conjecture that, under mild assumptions on $R, \varphi_{R}$ is eventually a quasi-polynomial when it is bounded by some polynomial.
\end{abstract}

Keywords: Relational structure, profile, graded algebra, Hilbert function, Hilbert series, polynomial growth.

*This work was done under the auspices of the Intas programme Universal algebra and Lattice theory, and supported by CMCU Franco-Tunisien "Outils mathématiques pour l'informatique". 


\section{Introduction}

This paper is about a counting function: the profile of a relational structure $R$ and its interplay with a graded connected commutative algebra associated with $R$, the age algebra of $R$.

Many natural counting functions are profiles. Several interesting examples come from permutation groups. For example, if $G$ is a permutation group on a set $E$, the function $\theta_{G}$ which counts for every integer $n$ the number of orbits of the action of $G$ on the $n$-element subsets of $E$, is a profile, the orbital profile of $G$. Groups whose orbital profile takes only finite values are called oligomorphic; their study, introduced by Cameron, is a whole research subject by itself [Cam90, Cam09]. If $G$ acts on $\{1, \ldots, k\}$, the Hilbert function of the subalgebra $\mathbb{K}[X]^{G}$ of the polynomials in $\mathbb{K}[X]:=\mathbb{K}\left[X_{1}, \ldots, X_{k}\right]$ which are invariant under the action of $G$ is a profile, and in fact an orbital profile. This fact led Cameron to associate a graded algebra $\mathbb{K} \mathcal{A}(R)$ to each relational structure $R$ [Cam97]; its main feature is that its Hilbert function coincides with the profile of $R$ as long as it takes only finite values. As it is well know, the Hilbert function of a graded commutative algebra $A$ is eventually a quasi-polynomial (hence bounded by some polynomial), provided that $A$ is finitely generated. The converse does not hold. Still, this leads us to conjecture that the profile $\varphi_{R}$ of a relational structure $R$ is eventually a quasi-polynomial when $\varphi_{R}$ is bounded by some polynomial (and the kernel of $R$ is finite).

This conjecture holds if $R$ is an undirected graph [BBSS09] or a tournament [BP10]. In this paper, we prove that it holds for any relational structure $R$ admitting a finite monomorphic decomposition. Its age algebra is (essentially) a graded subalgebra of a finitely generated polynomial algebra. It needs not be finitely generated (see Example A.4). Still, and this is our main result, the profile is eventually a quasi-polynomial whose degree is controlled by the dimension of the monomorphic decomposition of $R$ (Theorem 1.7). This result was applied in [BP10] to show that the above conjecture holds for tournaments.

Relational structures admitting a finite monomorphic decomposition are not so peculiar. Many familiar algebras, like invariant rings of permutation groups, rings of quasisymmetric polynomials, can be realized this way. We give many examples in Appendix A. Further studies, notably a characterization of these relational structures for which the age algebra is finitely generated are included in [PT12].

The study of the profile started in the seventies; see [Pou06] for a survey and Pou08] for more recent results. Our line of work is parallel to the numerous researches made in recent years about the behavior of counting functions for hereditary classes made of finite structures, like undirected graphs, posets, tournaments, ordered graphs, or permutations, which also enter into this frame; see Kla08a and Bol98 for a survey, and BBM07, BBSS09, MT04, AA05, AAB07, Vat11, KK03, Kla08b, BHV08, OP11. These classes are hereditary in the sense that they contain all induced structures of each of their members; in several instances, members of these classes are counted up to isomorphism and with respect to their size.

Results point out jumps in the behavior of these counting functions. Such jumps 
were for example announced for extensive hereditary classes in Pou80, with a proof for the jump from constant to linear published in [Pou81b. The growth is typically polynomial or faster than any polynomial, though not necessarily exponential (as indicates the partition function; see Example A.6). For example, the growth of an hereditary class of graphs is either polynomial or faster than the partition function [BBSS09]. In several instances, these counting functions are eventually quasi-polynomials, e.g. [BBSS09] for graphs and KK03 for permutations.

Klazar asked in his survey Kla08a how the two approaches relate. At first glance, the structures we consider are more general; however the classes we consider in this paper are more restrictive: the profile of a relational structure $R$ is the counting function of its age, and ages are hereditary classes structures which are up-directed by embeddability ([Fra54]). A priori, results on the behavior of counting functions of ages do not extend straightforwardly to hereditary classes. There is a notable exception: the study of hereditary classes with polynomially bounded profiles can indeed be reduced to that of ages, thanks to the following result:

Theorem 0.1. Consider an hereditary class $\mathfrak{C}$ of finite structures of fixed finite signature. If $\mathfrak{C}$ has polynomially bounded profile then it is a finite union of ages. Otherwise it contains an age with non polynomially bounded profile.

The proof is given in section 4 .

This paper is organized as follows. In Section 1 we recall the definitions and basic properties of relational structures, their profiles, and age algebras, and state our guiding problems. We introduce the key combinatorial notion of monomorphic decomposition of a relational structure, mention the existence of a unique minimal one (Proposition 1.6), and state our main theorem (Theorem 1.7) together with some other results. In Section 2, we study the properties of monomorphic decompositions and prove Proposition 1.6, while Section 3 contains the proof of Theorem 1.7. Analyzing lots of examples has been an essential tool in this exploration. Appendix A gathers them, with a description of their age algebras; see in particular Examples A.4, A.18, and A.19, and Proposition A.23. We urge the reader to start by browsing them, and to come back to them each time a new notion is introduced. The overview of the results in Table 1 (which includes results from [PT12]) may be of help as well.

\section{Acknowledgments}

A strong impulse to this research came from a paper by Garsia and Wallach [GW03] who proved that the ring of quasi-symmetric polynomials is Cohen-Macaulay. Most, but not all, of the results where previously announced at the FPSAC conference in honor of Adriano Garsia [PT05, at the 14th Symposium of the Tunisian Mathematical Society, held in Hammamet in 2006 [Pou06], and at ROGICS'08 [PT08].

We would like to thank the anonymous referee for a thorough report including many helpful suggestions for improvements. 


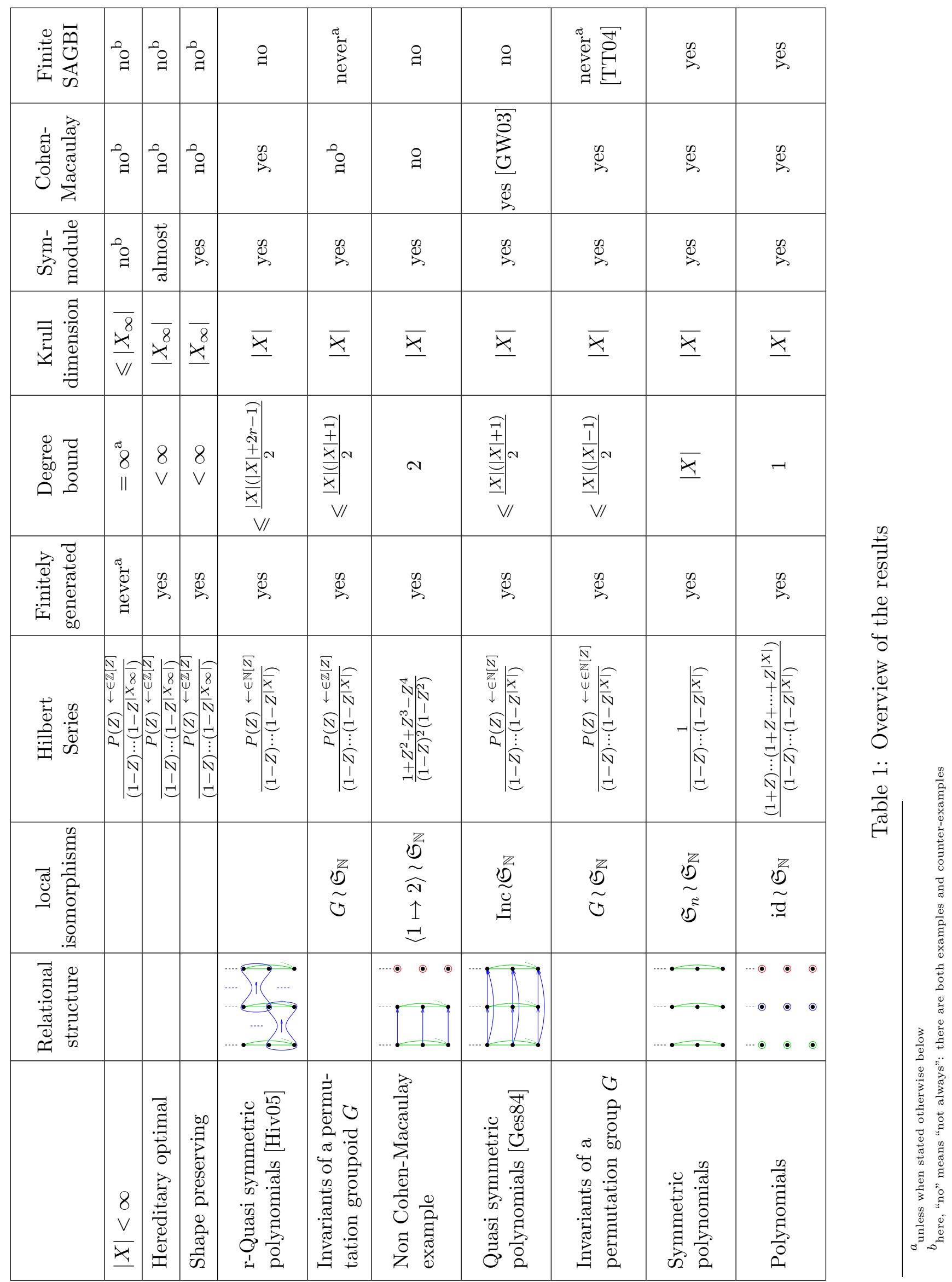




\section{Age algebras and quasi-polynomiality of relational structure admitting a finite monomorphic decom- position}

\subsection{Relational structures and their profile}

A relational structure is a realization of a language whose non-logical symbols are predicates. This is a pair $R:=\left(E,\left(\rho_{i}\right)_{i \in I}\right)$ made of a set $E$ and a family of $m_{i}$-ary relations $\rho_{i}$ on $E$. The set $E$ is the domain or base of $R$; the family $\mu:=\left(m_{i}\right)_{i \in I}$ is the signature of $R$; the signature is finite if $I$ is. The substructure induced by $R$ on a subset $A$ of $E$, simply called the restriction of $R$ to $A$, is the relational structure $R_{\uparrow A}:=\left(A,\left(A^{m_{i}} \cap \rho_{i}\right)_{i \in I}\right)$. The notion of isomorphism between relational structures is defined in the natural way. A local isomorphism of $R$ is any isomorphism between two restrictions of $R$. Two relational structures $R$ and $R^{\prime}$ are isomorphic is there is an isomorphism $f$ from $R$ onto $R^{\prime}$. We also say that they have the same isomorphism type. The isomorphism type of a relational structure is a formal object $\tau(R)$ such that a relational structure $R^{\prime}$ is isomorphic to $R$ if and only if $\tau\left(R^{\prime}\right)=\tau(R)$. In some situations, isomorphism types have a concrete representation. Let $R$ be as above. For two subsets $A$ and $A^{\prime}$ of $E$, we set $A \approx A^{\prime}$ if $R_{\uparrow A}$ and $R_{\uparrow A^{\prime}}$ are isomorphic. The orbit of a subset $A$ of $E$ is the set $\bar{A}$ of all subsets $A^{\prime}$ of $E$ such that $A^{\prime} \approx A$ (the name "orbit" is given by analogy with the case of permutation groups; see Subsection A.2). The orbit $\bar{A}$ can play the role of the isomorphism type $\tau(A):=\tau\left(R_{\uparrow A}\right)$ of $R_{\uparrow A}$.

The profile of $R$ is the function $\varphi_{R}$ which counts, for every integer $n$, the number $\varphi_{R}(n)$ of isomorphism types of restrictions of $R$ on $n$-element subsets. Clearly, this function only depends upon the set $\mathcal{A}(R)$ of isomorphism types of finite restrictions of $R$; this set, called the age of $R$, was introduced by R. Fraïssé (see [Fra00]). If the signature of $R$ is finite, $\varphi_{R}(n)$ is necessarily finite. In order to capture examples coming from algebra and group theory, we cannot preclude an infinite signature. However, since the profile is finite in these examples, and unless explicitly stated otherwise, we always make the assumption that $\varphi_{R}(n)$ is finite, even if the signature of $R$ is not.

The profile of an infinite relational structure is non-decreasing. Furthermore, provided some mild conditions, there are jumps in the behavior of the profile:

Theorem 1.1. Let $R:=\left(E,\left(\rho_{i}\right)_{i \in I}\right)$ be a relational structure on an infinite set. Then, $\varphi_{R}$ is non-decreasing. And provided that either the signature $\mu$ is bounded or the kernel $K(R)$ of $R$ is finite, the growth of $\varphi_{R}$ is either polynomial or as fast as every polynomial.

A map $\varphi: \mathbb{N} \rightarrow \mathbb{N}$ has polynomial growth, of degree $k$, if $a n^{k} \leqslant \varphi(n) \leqslant b n^{k}$ for some $a, b>0$ and $n$ large enough. The kernel of $R$ is the set $K(R)$ of $x \in E$ such that $\mathcal{A}\left(R_{\uparrow E \backslash\{x\}}\right) \neq \mathcal{A}(R)$. Relations with empty kernel are the age-inexhaustible relations of R. Fraïssé'(see [Fra00]). We call almost age-inexhaustible those with finite kernel.

The hypothesis about the kernel is not ad hoc. As it turns out, if the growth of the profile of a relational structure with a bounded signature is bounded by a polynomial then 
its kernel is finite. Some hypotheses on $R$ are needed, indeed for every increasing and unbounded map $\varphi: \mathbb{N} \rightarrow \mathbb{N}$, there is a relational structure $R$ such that $\varphi_{R}$ is unbounded and eventually bounded above by $\varphi$ (see [Pou81b]). The first part of the result was obtained in 1971 by the first author (see Exercise 8 p. 113 [Fra71]). A proof based on linear algebra is given in Pou76]. The second part was obtained in [Pou78] and a part was published in Pou81b.

The theorem above is not the best possible. It is natural to ask

Question 1.2. Does $\varphi_{R}$ have polynomial growth in the strong sense: $\varphi_{R}(n) \sim a n^{k}$ for some positive real $a$ whenever $R$ has bounded signature or finite kernel and $\varphi_{R}$ is bounded above by some polynomial.

This question, raised by Cameron Cam90] in the special case of orbital profile, is unsettled.

We consider a stronger condition. Recall that a $\operatorname{map} \varphi: \mathbb{N} \rightarrow \mathbb{N}$ is a quasi-polynomial of degree $k$ if $\varphi(n)=a_{k}(n) n^{k}+\cdots+a_{0}(n)$ whose coefficients $a_{k}(n), \ldots, a_{0}(n)$ are periodic functions. Note that, when a profile is eventually a quasi-polynomial, it has polynomial growth in the strong sense. Indeed, since the profile is non-decreasing, the coefficient $a_{k}(n)$ of highest degree of the quasipolynomial is constant. We make the following:

Conjecture 1.3. The profile of a relational structure with bounded signature or finite kernel is eventually a quasi-polynomial whenever the profile is bounded by some polynomial.

This conjecture is motivated by the association made by Cameron of a graded commutative algebra, the age algebra $\mathbb{K} \mathcal{A}(R)$, to a relational structure $R$. Indeed, if $A$ is a graded commutative algebra, the Hilbert function $h_{A}$ of $A$, where $h_{A}(n)$ is the dimension of the homogeneous component of degree $n$ of $A$, is eventually a quasi-polynomial whenever $A$ is finitely generated. In fact, if $A$ is such an algebra, the generating series $\mathcal{H}_{A}(Z):=\sum_{n} h_{A}(n) Z^{n}$, called the Hilbert series of $A$, is a rational fraction of the form

$$
\frac{P(Z)}{\left(1-Z^{n_{1}}\right)\left(1-Z^{n_{2}}\right) \cdots\left(1-Z^{n_{k}}\right)},
$$

where $1=n_{1} \leqslant \cdots \leqslant n_{k}$ and $P(Z) \in \mathbb{Z}[Z]$ (see e.g. CLO97, Chapter 9, $\left.\S 2\right]$ ). Furthermore, whenever $A$ is Cohen-Macaulay, $P(Z) \in \mathbb{N}[Z]$ for some choice of $n_{1}, \ldots, n_{k}$. And, as it is also well known ([Sta97]), a counting function is eventually a quasi-polynomial of degree at most $k-1$ whenever its generating series has the form (1) above.

As shown by Cameron, the Hilbert function of the age algebra of a relational structure $R$ is the profile of $R$ (provided that it takes only finite values). Thus, provided that the age algebra is finitely generated, Conjecture 1.3 and hence Question 1.2 have a positive answer. In fact, as we will see, there are many relational structures for which the generating series of their profile has the form (1) above, but few for which the age algebra is finitely generated.

We present now the age algebra. 


\section{$1.2 \quad$ Age algebras}

Let $\mathbb{K}$ be a field of characteristic 0 , and $E$ be a set. For $n \geqslant 0$, denote by $[E]^{n}$ the set of the subsets of $E$ of size $n$, and let $\mathbb{K}^{[E]^{n}}$ be the vector space of maps $f:[E]^{n} \rightarrow \mathbb{K}$. The set algebra is the graded connected commutative algebra $\mathbb{K}^{[E]^{<\omega}}:=\bigoplus_{n} \mathbb{K}^{[E]^{n}}$, where the product of $f:[E]^{m} \rightarrow \mathbb{K}$ and $g:[E]^{n} \rightarrow \mathbb{K}$ is defined as $f g:[E]^{m+n} \rightarrow \mathbb{K}$ such that:

$$
(f g)(A):=\sum_{\left(A_{1}, A_{2}\right)}: A_{A=A_{1} \uplus A_{2}} f\left(A_{1}\right) g\left(A_{2}\right) .
$$

Identifying a set $S$ with its characteristic function $\chi_{S}$, elements of the set algebra can be thought as (possibly infinite but of bounded degree) linear combination of sets; the unit is the empty set, and the product of two sets is their disjoint union, or 0 if their intersection is non trivial.

Let $R$ be a relational structure with base set $E$. A map $f:[E]^{m} \rightarrow \mathbb{K}$ is $R$-invariant if $f(A)=f\left(A^{\prime}\right)$ whenever $A \approx A^{\prime}$. It is easy to show that the product of two $R$-invariant maps is again invariant. The $\mathbb{K}$-vector space spanned by the $R$-invariant maps is therefore a graded connected commutative subalgebra of the set algebra, the age algebra of $R$, that we denote by $\mathbb{K} \mathcal{A}(R)$. It can be shown that two relational structures with the same age yield the same algebra (up to an isomorphism of graded algebras); thus the name, coined by Cameron who invented the notion [Cam97]. If the profile of $R$ takes only finite values, then $\mathbb{K} \mathcal{A}(R)$ identifies with the set of (finite) linear combinations of elements of $\mathcal{A}(R)$ and, as pointed out by Cameron, $\varphi_{R}(n)$ is the dimension of the homogeneous component of degree $n$ of $\mathbb{K} \mathcal{A}(R)$; indeed, define an orbit sum as the characteristic function of an orbit; more specifically, the orbit sum of an isomorphism type $\tau \in \mathcal{A}(R)$ is the characteristic

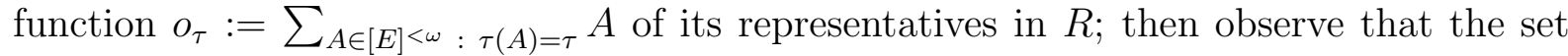
of orbit sums form a basis of the age algebra $\mathbb{K} \mathcal{A}(R))$. By a slight abuse, we sometimes identify $\tau$ with its orbit sum to see it as an element of $\mathbb{K} \mathcal{A}(R)$.

Given three isomorphic types $\tau, \tau_{1}, \tau_{2}$, we define a coefficient $c_{\tau_{1}, \tau_{2}}^{\tau}$ by taking any subset $A$ of $E$ of type $\tau$ and setting

$$
c_{\tau_{1}, \tau_{2}}^{\tau}:=\left|\left\{\left(A_{1}, A_{2}\right): A_{1} \uplus A_{2}=A, \tau\left(A_{1}\right)=\tau_{1}, \tau\left(A_{2}\right)=\tau_{2}\right\}\right| .
$$

Clearly, this coefficient does not depend on the choice of $A$. The collection $\left(c_{\tau_{1}, \tau_{2}}^{\tau}\right)_{\tau, \tau_{1}, \tau_{2}}$ are the structure constants of the age algebra:

$$
o_{\tau_{1}} o_{\tau_{2}}=\sum_{\tau} c_{\tau_{1}, \tau_{2}}^{\tau} o_{\tau}
$$

Let us illustrates the role that the age algebra of Cameron can play. Let $e:=\sum_{a \in E}\{a\}$, which we can think of as the sum of isomorphic types of the one-element restrictions of $R$. Let $U$ be the graded free algebra $\mathbb{K}[e]=\bigoplus_{n=0}^{\infty} \mathbb{K} e^{n}$. Cameron (see [Cam97]) proved:

Theorem 1.4. If $R$ is infinite then e is not a zero-divisor; namely for any $u \in \mathbb{K} \mathcal{A}(R)$, $e u=0$ if and only if $u=0$. 
This result implies that $\varphi_{R}$ is non decreasing. Indeed, the image of a basis of the vector space $\mathbb{K} \mathcal{A}(R)_{n}$ under multiplication by $e$ is a linearly independent subset of $\mathbb{K} \mathcal{A}(R)_{n+1}$.

The relationship between profile and age algebra is particularly simple for relational structures with bounded profile. These structures were characterized in [FP71] for finite signature and in [Pou81b, Théorème 1.2] for arbitrary signature, by means of Ramsey theorem. We recall it below.

Let $R:=\left(E,\left(\rho_{i}\right)_{i \in I}\right)$ be a relational structure, $F$ be a subset of $E$ and $B:=E \backslash F$. Following R. Fraïssé, who invented these notions, we say that $R$ is $F$-monomorphic if for every integers $n$ and every $A, A^{\prime} \in[B]^{n}$ there is an isomorphism from $R_{\uparrow A \cup F}$ onto $R_{\uparrow A^{\prime} \cup F}$ which is the identity on $F$. We say that $R$ is $F$-chainable if there is a linear order $\leqslant$ on $B$ such that the local isomorphisms of $(B, \leqslant)$ extended by the identity on $F$ are local isomorphisms of $R$. We say that $R$ is almost monomorphic, resp. almost chainable, if $R$ is $F$-monomorphic, resp. $F$-chainable, for some finite subset $F$ of $E$. We say that $R$ is monomorphic, resp. chainable, if one can take $F$ empty.

Assume for example, that $R$ is made of a single relation $\rho$ and is chainable by some linear order $\leqslant$. If $\rho$ is unary, then it is the full or empty relation. If $\rho$ is binary, there are eight possibilities: four if $\rho$ is reflexive ( $\rho$ coincides with either $\leqslant$, its opposite $\geqslant$, the equality relation $=$, or the complete relation $E \times E$ ) and four if not (the same as for the reflexive case, with loops removed).

For infinite relational structures, the notions of monomorphy and chainability coincide [Fra54]. For finite structures, they are distinct. However, it was proved by C. Frasnay that, for any integer $n$, there is an integer $f(n)$ such that every monomorphic relational structure of arity at most $n$ and size at least $f(n)$ is chainable (see [Fra65] and [Fra00, Chapter 13]).

The following theorem links the profile with the age algebra in the context of almostchainable and almost-monomorphic relational structures.

Theorem 1.5. Let $R$ be a relational structure with E infinite. Then, the following properties are equivalent:

(a) The profile of $R$ is bounded.

(b) $R$ is almost-monomorphic.

(c) $R$ is almost-chainable.

(d) The Hilbert series is of the following form, with $P(Z) \in \mathbb{N}[Z]$ and $P(1) \neq 0$ :

$$
\mathcal{H}_{R}=\frac{P(Z)}{1-Z}
$$

(e) The age algebra is a finite dimensional free-module over the free-algebra $\mathbb{K}[e]$, where $e:=\sum_{a \in E} a$; in particular it is finitely generated and Cohen-Macaulay. 
Proof. Trivially, (c) implies (b) and (b) implies (a). The equivalence between (a) and (c) is in [FP71] for finite signature and in [Pou81b, 1.2 Théorème, p. 317] for arbitrary signature.

Straightforwardly, (e) implies (d) and (d) implies (a). Finally (e) follows from (a): indeed, by Theorem 1.4, $e$ is not a zero divisor in $\mathbb{K} \mathcal{A}(R)$; using the grading, it follows that $\mathbb{K}[e]$ is a free algebra and that $\mathbb{K} \mathcal{A}(R)$ is a free-module over $\mathbb{K}[e]$. Since the profile is bounded, this free-module is finite dimensional.

\subsection{Relational structures admitting a finite monomorphic de- composition}

We now introduce a combinatorial notion which generalizes that of almost-monomorphic relational structure.

Let $E$ be a set and $\left(E_{x}\right)_{x \in X}$ be a set partition of $E$. Write $X_{\infty}:=\left\{x \in X:\left|E_{x}\right|=\infty\right\}$. For a subset $A$ of $E$, set $d_{x}(A):=\left|A \cap E_{x}\right|, d(A):=\left(d_{x}(A)\right)_{x \in X}$ and $\bar{d}(A)$ be the sequence $d(A)$ sorted in decreasing order. The sequence $\bar{d}(A)$ is the shape of $A$ with respect to the partition $\left(E_{x}\right)_{x \in X}$. When $A$ is finite it is often convenient, and even meaningful, to encode

$d(A)$ as a monomial in $\mathbb{K}[X]$, namely $X^{A}:=\prod_{x \in X} x^{d_{x}(A)}$; furthermore, up to trailing zero parts, $\bar{d}(A)$ is an integer partition of $|A|$.

Let $R$ be a relational structure on $E$. We call $\left(E_{x}\right)_{x \in X}$ a monomorphic decomposition of $R$ if the induced structures on two finite subsets $A$ and $A^{\prime}$ of $E$ are isomorphic whenever $X^{A}=X^{A^{\prime}}$. The following proposition states the basic result about monomorphic decompositions; we will prove it in a slightly more general setting (see Proposition 2.12).

Proposition 1.6. There is a monomorphic decomposition of $R$ of which every other monomorphic decomposition of $R$ is a refinement.

This monomorphic decomposition is called minimal; its number $k:=k(R):=\left|X_{\infty}\right|$ of infinite blocks is the monomorphic dimension of $R$.

Our main result, which we prove in Section 3, is a complete solution for Conjecture 1.3 for relational structures admitting a finite monomorphic decomposition (the almost-monomorphic ones being those of dimension 1 ):

Theorem 1.7. Let $R$ be an infinite relational structure with a finite monomorphic decomposition, and let $k$ be its monomorphic dimension. Then, the generating series $\mathcal{H}_{R}$ is a rational fraction of the following form, with $P \in \mathbb{Z}[Z]$ and $P(1) \neq 0$ :

$$
\frac{P(Z)}{(1-Z)\left(1-Z^{2}\right) \cdots\left(1-Z^{k}\right)}
$$

In particular, $\varphi_{R}$ is eventually a quasi polynomial of degree $k-1$, hence $\varphi_{R}(n) \sim a n^{k-1}$.

This refines Theorem 2.16 of [PT05], by refining the denominator and the growth rate.

Among relational structures which admit a finite monomorphic decomposition, those made of a single unary or binary relation are easy to characterize (cf. Corollary 2.26). For 
example, the undirected graphs admitting a finite monomorphic decomposition are the lexicographical sums of cliques or independent sets indexed by a finite graph. Similarly, the tournaments admitting a finite monomorphic decomposition are the lexicographical sums of acyclic (aka transitive) tournaments indexed by a finite tournament. In Section 2.3 we prove the following generalization.

Theorem 1.8. A relational structure $R:=\left(E,\left(\rho_{i}\right)_{i \in I}\right)$ admits a finite monomorphic decomposition if and only if there exists a linear order $\leqslant$ on $E$ and a finite partition $\left(E_{x}\right)_{x \in X}$ of $E$ into intervals of $(E, \leqslant)$ such that every local isomorphism of $(E, \leqslant)$ which preserves each interval is a local isomorphism of $R$. Moreover, there exists such a partition whose number of infinite blocks is the monomorphic dimension of $R$.

The interest for this notion goes beyond these examples and this characterization. It turns out that familiar algebras like invariant rings of finite permutation groups can be realized as age algebras of relational structures admitting a finite monomorphic decomposition (see Example A.16); another example is the algebra of quasi-symmetric polynomials and variants (see Examples A.18 of Appendix A.

The relationship with polynomials is not accidental:

Proposition 1.9 ([PT12 $])$. If $R$ admits a monomorphic decomposition into finitely many blocks $E_{1}, \ldots, E_{k}$, all infinite, then the age algebra $\mathbb{K} \mathcal{A}(R)$ is isomorphic to a subalgebra $\mathbb{K}\left[x_{1}, \ldots, x_{k}\right]^{R}$ of the algebra $\mathbb{K}\left[x_{1}, \ldots, x_{k}\right]$ of polynomials in the indeterminates $x_{1}, \ldots, x_{k}$.

Invariant rings of finite permutation groups, as well as the algebras of quasi-symmetric polynomials are finitely generated and in fact Cohen-Macaulay. It is however worth noticing on the onset that there are examples of relational structures such that

- $X$ is finite, but the age algebra is not finitely generated (see Example A.4);

- $X$ is finite, the Hilbert series is of the form of Equation 1 with $P(Z) \in \mathbb{N}[Z]$ but the age algebra is not Cohen-Macaulay (see Example A.19;

- $X$ is infinite but the profile still has polynomial growth (see Examples A.9 and A.10).

In those examples, the profile is still a quasi-polynomial. This raises the following problems.

Problems 1.10. Let $R$ be a relational structure whose profile is bounded by some polynomial. Find combinatorial conditions on $R$ for

(a) the profile to be eventually a quasi-polynomial;

(b) the age algebra to be finitely generated;

(c) the age algebra to be Cohen-Macaulay.

We give here a partial answer to (a), while (b) and (c) are investigated in length in a subsequent paper [PT12]. 


\section{Monomorphic decompositions}

We start by stating some simple properties of set partitions. For the sake of completeness, proofs are included. Then, we apply those results to the lattice of monomorphic decompositions (Proposition 2.12), and derive a lower bound on the profile (Theorem 2.17)

\subsection{On certain lattices of set partitions}

Fix a set $E$, finite or not, and a collection $\mathcal{C}$ of subsets of $E$ which are called good. We assume that this collection satisfies the following:

Axioms 2.1 (Goodness axioms). (a) Singletons are good;

(b) A subset of a good subset is good;

(c) A (possibly infinite) union of good subsets with a non trivial intersection is good.

Lemma 2.2. A collection $\mathcal{C}$ of subsets of $E$ satisfies Axioms 2.1 if and only if every subset of a maximal member of $\mathcal{C}$ belongs to $\mathcal{C}$ and the maximal members of $\mathcal{C}$ form a set partition of $E$.

Proof. Suppose that $\mathcal{C}$ satisfy Axioms 2.1 above. The fact that subsets of maximal members of $\mathcal{C}$ belong to $\mathcal{C}$ follows directly from (b). Now take some $a \in E$. By (a) and (c), the union $\mathcal{C}(a)$ of all good subsets containing $a$ is good. Furthermore, any maximal good subset of $E$ (for inclusion) is of this form. Using (c), two maximal good subsets $\mathcal{C}(a)$ and $\mathcal{C}(b)$ either coincide or are disjoint. Hence, the collection $\mathcal{P}(\mathcal{C}):=\{\mathcal{C}(a)\}_{a \in E}$ forms a set partition of $E$ into good subsets. The converse is immediate.

Let $\mathcal{C}$ be a collection of subsets of $E$ satisfying Axioms 2.1. The components of $\mathcal{C}$ are the maximal members of $\mathcal{C}$. We denote by $\mathcal{P}(\mathcal{C})$ the partition of $E$ into components of $\mathcal{C}$ and by $s(\mathcal{C})$ the number of components, that is the size of $\mathcal{P}(\mathcal{C})$.

We consider the refinement order on the set $\mathcal{S}(E)$ of the set partitions on $E$, choosing by convention that $\mathcal{P} \preceq \mathcal{Q}$ if $\mathcal{P}$ is coarser than $\mathcal{Q}$ (that is each block of $\mathcal{P}$ is a union of blocks of $\mathcal{Q})$. With this ordering $\mathcal{S}(E)$ forms a lattice, with $\{E\}$ as minimal element and $\{\{a\}: a \in E\}$ as maximal element.

A set partition $\mathcal{P}:=\left(P_{x}\right)_{x \in X}$ of $E$ is good if each block $P_{x}$ is good. For example, $\mathcal{P}(\mathcal{C})$ is good and so is the trivial partition into singletons. From Lemma 2.2 above we have:

Proposition 2.3. A set partition $\mathcal{P}$ is good if and only if it refines $\mathcal{P}(\mathcal{C})$. Hence the set of all good set partitions is a principal filter of the lattice $\mathcal{S}(E)$; in particular, it is stable under joins and meets.

Due to this fact, we name $\mathcal{P}(\mathcal{C})$ the minimal good set partition.

Take now a set $E$, and associate to each subset $D$ of $E$ a collection $\mathcal{C}_{D}$ of subsets of $D$, called $D$-good, and satisfying the axioms above. Assume further that the following axiom is satisfied: 
Axiom 2.4 (Goodness axioms, continued). (d) If $D_{1} \subseteq D_{2} \subseteq E$ and $F \subseteq D_{2}$ is $D_{2}$-good, then $F \cap D_{1}$ is $D_{1}$-good.

Take $D_{1} \subseteq D_{2}$. A set partition $\mathcal{P}$ of $D_{2}$ induces (by intersection of each of its block with $D_{1}$ and removal of the empty ones) a set partition of $D_{1}$. The latter is properly induced by $\mathcal{P}$ if $D_{1}$ intersects non trivially each block of $\mathcal{P}$ (that is no block vanishes in the process).

Axiom (d) implies right away the following properties.

Proposition 2.5. Let $D_{1} \subseteq D_{2} \subseteq D_{3} \subseteq E$.

(a) Any $D_{2}$-good set partition of $D_{2}$ induces a $D_{1}$-good set partition of $D_{1}$.

(b) The size of the minimal good set partition of $D_{2}$ is at least that of the minimal good set partition of $D_{1}$. If it is finite there is equality if and only if the minimal good set partition $\mathcal{P}\left(D_{1}\right)$ of $D_{1}$ is properly induced by $\mathcal{P}\left(D_{2}\right)$.

(c) If $\mathcal{P}\left(D_{1}\right)$ is properly induced by $\mathcal{P}\left(D_{3}\right)$, then $\mathcal{P}\left(D_{1}\right)$ is properly induced by $\mathcal{P}\left(D_{2}\right)$ which itself is properly induced by $\mathcal{P}\left(D_{3}\right)$

To derive the main desired property, we need to add a last axiom on good subsets.

Axiom 2.6 (Goodness axioms, continued). (e) If $\mathcal{D}$ is a chain of subsets of $E$ whose union is $D$, then a subset $F$ of $D$ is $D$-good as soon as $F \cap D^{\prime}$ is $D^{\prime}$-good for all $D^{\prime} \in \mathcal{D}$.

Lemma 2.7. Every subset $D$ of $E$ such that $s\left(\mathcal{C}_{D}\right)$ is finite includes some finite subset $F$ such that $s\left(\mathcal{C}_{F}\right)=s\left(\mathcal{C}_{D}\right)$.

Proof. We argue by induction on the cardinality $\kappa$ of $D$. If $\kappa$ is finite there is nothing to prove. Suppose otherwise that $\kappa$ is infinite, and write $D$ as the union of a chain $\mathcal{D}$ of subsets $D^{\prime}$ of $D$, such that $\left|D^{\prime}\right|<\kappa$. By induction, we may assume that the property holds for each $D^{\prime}$ in $\mathcal{D}$. There remains to prove that $s\left(\mathcal{C}_{D^{\prime}}\right)=s\left(\mathcal{C}_{D}\right)$ for some $D^{\prime} \in \mathcal{D}$. According to Proposition 2.5 (a) we have $s\left(\mathcal{C}_{D^{\prime}}\right) \leqslant s\left(\mathcal{C}_{D^{\prime \prime}}\right) \leqslant s\left(\mathcal{C}_{D}\right)$ for all $D^{\prime} \subseteq D^{\prime \prime} \subseteq D$. Since $s\left(\mathcal{C}_{D}\right)$ is finite, there is some $k$ and some $D^{\prime} \in \mathcal{D}$ such that $s\left(\mathcal{C}_{D^{\prime \prime}}\right)=k$ for all $D^{\prime \prime} \in \mathcal{D}$ such that $D^{\prime} \subset D^{\prime \prime}$. Set $X:=\{1, \ldots, k\}$. For each such $D^{\prime \prime}$, denote $\left(D_{x}^{\prime \prime}\right)_{x \in X}$ the minimal good set partition $\mathcal{P}\left(\mathcal{C}_{D^{\prime \prime}}\right)$ of $D^{\prime \prime}$. Without loss of generality, and up to some renumbering of the blocks, we may assume that, for each $x \in X$, the set $\mathcal{D}_{x}:=\left\{D_{x}^{\prime \prime}: D^{\prime} \subseteq D^{\prime \prime} \in \mathcal{D}\right\}$ forms a chain for inclusion. Using Axiom (e), the union $D_{x}$ of those is a $D$-good block. Then, $\left(D_{x}\right)_{x \in X}$ is a good set partition of $D$ into $k$ blocks and thus it is the minimal one, proving that $s\left(\mathcal{C}_{D^{\prime}}\right)=k=s\left(\mathcal{C}_{D}\right)$, as desired.

Proposition 2.8. Assume that $E$ has a good partition into finitely many blocks, and let $\mathcal{F}$ be the collection of all subsets $D$ of $E$ whose minimal good set partition is properly induced by that of $E$. Then, a subset $D$ is in $\mathcal{F}$ if and only if it includes a finite subset in $\mathcal{F}$.

Proof. The "if" part follows from Proposition 2.5. The converse follows from Lemma 2.7. 


\subsection{Minimal monomorphic decompositions}

We start with some elementary properties of the monomorphic decompositions of a relational structure $R$ with base set $E$, and in particular we show that their blocks satisfy the goodness axioms.

Lemma 2.9. Let $R$ be a relational structure with base set $E$. For a subset $F$ of $E$, the following conditions are equivalent:

(i) there exists some monomorphic decomposition of $R$ admitting $F$ as a block;

(ii) the set partition $\{F\} \cup\{\{x\}\}_{x \notin F}$ is a monomorphic decomposition of $R$;

(iii) for every pair $A, A^{\prime}$ of subsets of $E$ with the same finite cardinality the induced structures on $A$ and $A^{\prime}$ are isomorphic whenever $A \backslash F=A^{\prime} \backslash F$.

A subset $F$ is a monomorphic part of $R$ if any (and therefore all) of the previous conditions hold.

Note that in (iii) above, we do not not impose that the induced substructures on $A$ and $A^{\prime}$ are isomorphic via an isomorphism which is the identity on $A \backslash F$. However, this condition is fulfilled as soon as $F$ is an infinite monomorphic part which is maximal w.r.t. inclusion (see Theorem 2.25, beware of a different use of the letter $F$ in this result).

Lemma 2.10. A set partition $\left(E_{x}\right)_{x \in X}$ of $E$ is a monomorphic decomposition of $R$ if and only if it is made of monomorphic parts of $R$.

Proof. The "only if" part is by definition. Consider now a set partition whose blocks are monomorphic parts of $R$, and take $A$ and $A^{\prime}$ such that $X^{A}=X^{A^{\prime}}$. Let $E_{1}, \ldots, E_{k}$ be the blocks that $A$ and $A^{\prime}$ intersect non trivially, and for $l \in 0, \ldots, k$, set $A_{0}:=A^{\prime}$, $A_{l}:=\left(A \cap E_{1}\right) \cup \cdots \cup\left(A \cap E_{l}\right) \cup\left(A^{\prime} \cap E_{l+1}\right) \cup \cdots \cup\left(A^{\prime} \cap E_{k}\right)$. Then, for $l<k, A_{l}$ and $A_{l+1}$ have the same cardinality and $A_{l} \backslash E_{l+1}=A_{l+1} \backslash E_{l+1}$ are equal. Since $A_{0}=A^{\prime}$ and $A_{k}=A$, one has $A \approx A^{\prime}$, as desired.

Lemma 2.11. Let $R$ be a relational structure on a set $E$. Then the set of monomorphic parts of $R$ satisfies Axioms 2.1. If furthermore for every subset $D$ of $E$ the good sets of $D$ consist of the monomorphic parts of $R_{\uparrow D}$ then Axioms 2.4, and 2.6 are satisfied.

Proof. We denote by $A$ and $A^{\prime}$ two subsets of $E$ of the same finite cardinality.

Axiom 2.1(a): if $F$ is a singleton, and $A \backslash F=A^{\prime} \backslash F$, then $A$ and $A^{\prime}$ are equal, hence trivially isomorphic.

Axiom 2.1(b): let $F$ be a monomorphic part of $R$ and $F^{\prime} \subseteq F$. If $A \backslash F^{\prime}=A^{\prime} \backslash F^{\prime}$, then $A \backslash F=A^{\prime} \backslash F$, and therefore $A$ and $A^{\prime}$ are isomorphic.

Axiom 2.1(c): let $\left(F_{i}\right)_{i \in I}$ be a (possibly infinite) family of monomorphic parts which all share at least a common point $x$, and set $F=\bigcup_{i \in I} F_{i}$, and assume $A \backslash F=A^{\prime} \backslash F$.

Without loss of generality, we may assume that $A$ and $A^{\prime}$ differ by a single point: $A=B \cup\{a\}$ and $A^{\prime}=B \cup\left\{a^{\prime}\right\}$ with $a$ and $a^{\prime}$ in $F$ (otherwise, pickup a sequence 
$A:=A_{1}, \ldots, A_{l}=A^{\prime}$, where at each step $A_{j} \backslash F=A \backslash F$, and $A_{j}$ and $A_{j+1}$ differ by a single point; then use transitivity).

If $\left\{a, a^{\prime}\right\}$ is a subset of some $F_{i}$, then it forms a monomorphic part. Using that $A \backslash\left\{a, a^{\prime}\right\}=B=A^{\prime} \backslash\left\{a, a^{\prime}\right\}$, wet get $A \approx A^{\prime}$.

Otherwise, take $F_{i}$ and $F_{i^{\prime}}$ such that $a \in F_{i}$ and $a^{\prime} \in F_{i}^{\prime}$.

If $x \notin B$, then using successively that $\{a, x\} \subseteq F_{i}$ is a monomorphic part and $\left\{a^{\prime}, x\right\} \subseteq$ $F_{i}^{\prime}$ is a monomorphic part, one gets,

$$
A=B \cup\{a\} \approx B \cup\{x\} \approx B \backslash \cup\left\{a^{\prime}\right\}=A^{\prime} .
$$

Similarly, if $x \in B$,

$$
A=B \cup\{a\} \approx B \backslash\{x\} \cup\left\{a, a^{\prime}\right\} \approx B \backslash \cup\left\{a^{\prime}\right\}=A^{\prime} .
$$

Axiom 2.4(d): Let $F$ be $D_{2}$-good, and assume that $A$ and $A^{\prime}$ are subsets of $D_{1}$ such that $A \backslash\left(F \cap D_{1}\right)=A^{\prime} \backslash\left(F \cap D_{1}\right)$. Then, $A \backslash F=A \backslash\left(F \cap D_{1}\right)=A^{\prime} \backslash\left(F \cap D_{1}\right)=A^{\prime} \backslash F$; hence $A \approx A^{\prime}$.

Axiom 2.6(e): assume $A$ and $A^{\prime}$ are subsets of $D$ such that $A \backslash F=A \backslash F$. Take $D^{\prime}$ large enough in $\mathcal{D}$ so that $D^{\prime}$ contains both $A$ and $A^{\prime}$. Then $A \backslash\left(F \cap D^{\prime}\right)=A \backslash F=A^{\prime} \backslash F=$ $A^{\prime} \backslash\left(F \cap D^{\prime}\right)$, and therefore $A \approx A^{\prime}$.

We can now specialize Proposition 2.3 to monomorphic decompositions, to prove and refine Proposition 1.6.

Proposition 2.12. The maximal monomorphic parts of $R$ form a monomorphic decomposition of $R$. Furthermore, the other monomorphic decompositions are exactly the finer set partitions of this partition.

The monomorphic components of $R$, or components for short, are the maximal monomorphic parts of $R$. We denote by $c(R)$ the number of components of $R$. The partition of $E$ into components, that we denote by $\mathcal{P}(R)$, is the minimal monomorphic decomposition of $R$.

The main consequence of Proposition 2.12 is the following:

Corollary 2.13. Let $R$ and $R^{\prime}$ be two relational structures with domains $E$ and $E^{\prime}$ respectively. If $R$ and $R^{\prime}$ are isomorphic, then the components of $R$ are mapped bijectively onto the components of $R^{\prime}$ by any isomorphism $\sigma$. In particular, every automorphism of $R$ induces a permutation of the components of $R$ and, if the domain $E$ of $R$ is finite, $E$ and $E^{\prime}$ have the same number of components and the same shape w.r.t. their minimal decompositions.

Propositions 2.5 and 2.8 also apply, and will be used in the sequel. In particular, we get the following:

Proposition 2.14. Let $R$ be a relational structure and $R^{\prime}$ be a restriction of it. Then any monomorphic decomposition of $R$ induces a monomorphic decomposition of $R^{\prime}$. Note however that this monomorphic decomposition may have fewer components, and that minimality is not necessarily preserved. 
Let $\left(E_{x}\right)_{x \in X}$ be a partition of a set $E$. Given $d \in \mathbb{N}$, call $d$-fat a subset $A$ of $E$ such that, for all $x \in X, d_{x}(A) \geqslant d$ whenever $A \nsupseteq E_{x}$. We prove that, if the minimal monomorphic decomposition has finitely many components, then the isomorphism relation is shape preserving on fat enough sets, and we derive a lower bound on the profile.

Lemma 2.15. Let $R$ be an infinite relational structure on a set $E$ admitting a finite monomorphic decomposition. Then, there exists some integer $d$ such that on every $d$-fat subset $A$ of $E$ (w.r.t. $\mathcal{P}(R))$ the partition $\mathcal{P}(R)_{\uparrow A}$ induced by $\mathcal{P}(R)$ on $A$ coincides with $\mathcal{P}\left(R_{\uparrow A}\right)$. In particular the shape of $A$ w.r.t. $\mathcal{P}(R)$ coincides with the shape of $A$ w.r.t. $\mathcal{P}\left(R_{\uparrow A}\right)$.

Proof. Let $E$ be the base set of $R$ and $\left(E_{x}\right)_{x \in X}$ be the minimal partition of $E$ into monomorphic parts. According to Proposition 2.8 there is a family $\mathcal{F}$ of finite subsets $F$ of $E$ such that for every subset $D$ of $E$ the minimal partition of $D$ into monomorphic parts of $R_{\lceil D}$ is properly induced by the partition of $E$ if and only if $D$ contains some member of $\mathcal{F}$. Pick $F \in \mathcal{F}$. We claim that every subset $F^{\prime}$ of $E$ such that $d\left(F^{\prime}\right)=d(F)$ has the same property as $F$. Indeed, according to (c) of Proposition 2.5, it suffices to prove that $c\left(R_{\uparrow F^{\prime}}\right)=c(R)$. This fact is straightforward, indeed, since $d\left(F^{\prime}\right)=d(F), R_{\uparrow F}$ is isomorphic to $R_{\mid F^{\prime}}$, hence from Corollary 2.13 the components of $R_{\uparrow F}$ correspond bijectively to the components of $R_{\uparrow F^{\prime}}$. Hence, $c\left(\overline{R_{\uparrow F}}\right)=c\left(R_{\uparrow F^{\prime}}\right)$. By our choice of $F, c\left(R_{\uparrow F}\right)=c(R)$. Since the components of $R$ induce a monomorphic decomposition of $R_{\left\lceil F^{\prime}\right.}, c\left(R_{\uparrow F^{\prime}}\right) \leqslant c(R)$. This yields $c\left(R_{\mid F^{\prime}}\right)=c(R)$, as required. Let $d:=\max \left\{\left|F \cap E_{x}\right|: x \in X\right\}$. Let $A$ be a $d$-fat subset of $E$ w.r.t. the partition $\left(E_{x}\right)_{x \in X}$. Then $A$ contains a subset $F^{\prime}$ such that $d\left(F^{\prime}\right)=d(F)$ and we are done.

With the notations of Lemma 2.15 we have

Corollary 2.16. Two isomorphic d-fat subsets of $E$ always have the same shape w.r.t. $\mathcal{P}(R)$.

Theorem 2.17. Let $R$ be an infinite relational structure with a finite monomorphic decomposition, and let $k$ be its monomorphic dimension. Then, the profile $\varphi_{R}$ of $R$ is bounded from below by a polynomial of degree $k-1$; namely, there exists $n_{0}$ such that for $n \geqslant n_{0}, \varphi_{R}(n) \geqslant \wp_{k}\left(n-n_{0}\right)$, where $\wp_{k}(m)$ is the number of integer partitions of $m$ in at most $k$ parts.

Proof. Take $d$ as in Lemma 2.15, and let $n_{0}:=k_{1} d+m$, where $k_{1}$ is the number of monomorphic components of $R$ having at least $d$ elements, and $m$ is the sum of the cardinalities of the other finite monomorphic components of $R$.

Let $E_{1}, \ldots, E_{p}$ be the monomorphic components of $R$, enumerated in such a way that $\left|E_{1}\right| \geqslant \cdots \geqslant\left|E_{p}\right|$. To each decreasing sequence $x:=x_{1} \geqslant \cdots \geqslant x_{k^{\prime}}$ of positive integers such that $k^{\prime} \leqslant k$ and $x_{1}+\cdots+x_{k^{\prime}}=n-n_{0}$ associate an $n$-element subset $A(x)$ of $E$ such that $\left|A(x) \cap E_{i}\right|$ is respectively $d+x_{i}$ if $i \leqslant k^{\prime}, d$ if $k^{\prime}<i \leqslant k_{1}$ and $\left|E_{i}\right|$ if $k_{1}<i \leqslant p$. The set $A(x)$ is $d$-fat and its shape is $\left(\left|A(x) \cap E_{1}\right|, \ldots,\left|A(x) \cap E_{p}\right|\right)$. Clearly, if $x$ and $x^{\prime}$ are two distinct sequences as above, the shapes of $A(x)$ and $A\left(x^{\prime}\right)$ are distincts. Since these sets 
are $d$-fats, the restrictions $R_{\uparrow A(x)}$ and $R_{\uparrow A\left(x^{\prime}\right)}$ are not isomorphic. The claimed inequality follows.

As it is well known, $\wp_{k}(m)$ is asymptotically equivalent to $\frac{m^{k-1}}{(k-1) ! k !}$ (see e.g. VLW92]). Thus $\varphi_{R}(n)$ is asymptotically bounded below by $\frac{n^{k-1}}{(k-1) ! k !}$.

\subsection{Monomorphic decompositions, chainability and a proof of Theorem 1.8}

In Theorem 2.25 below, we present the relationship between the notions of chainability, monomorphy and our notion of monomorphic parts. The key ingredients of the proof are Ramsey's theorem, Compactness theorem of first order logic and some properties of the kernel of relational structures. We give below the facts we need.

Let $R:=\left(E,\left(\rho_{i}\right)_{i \in I}\right)$ a relational structure. For each subset $I^{\prime}$ of $I$ we set $R^{I^{\prime}}:=$ $\left(E,\left(\rho_{i}\right)_{i \in I^{\prime}}\right)$.

Compactness theorem of first order logic yields the following lemma:

Lemma 2.18. A relational structure $R:=\left(E,\left(\rho_{i}\right)_{i \in I}\right)$ is $F$-chainable if and only if for each finite subset $F^{\prime}$ of $F$ and every finite subset $I^{\prime}$ of $I, R_{\uparrow(E \backslash F) \cup F^{\prime}}^{I^{\prime}}$ is $F^{\prime}$-chainable.

Ramsey's theorem yields the following result:

Lemma 2.19 (Fraïssé [Fra54]). Let $R$ be a relational structure with domain $E$, and $F$ be a finite subset of $E$. If the signature of $R$ is finite then there is an infinite subset $E^{\prime}$ of $E$ containing $F$ on which the restriction $R^{\prime}:=R_{\uparrow E^{\prime}}$ is $F$-chainable.

We also need some properties of the kernel. Most of these properties are based on the following simple lemma (see [Pou79] for finite signature and [PS01, 3 of Lemma 2.12] for the general case).

Lemma 2.20. For all $a, b \in E, \mathcal{A}\left(R_{\lceil E \backslash\{a\}}\right)=\mathcal{A}(R) \Longrightarrow \mathcal{A}\left(R_{\lceil E \backslash\{a, b\}}\right)=\mathcal{A}\left(R_{\lceil E \backslash\{b\}}\right)$.

From this property, we have easily:

Lemma 2.21. Let $R$ be a relational structure with domain $E$, and $E^{\prime}$ be a subset of $E$. If $K(R) \subseteq E^{\prime}$ and $E \backslash E^{\prime}$ is finite then $R$ and $R_{\uparrow E^{\prime}}$ have the same age and the same kernel.

Lemma 2.22. Let $R$ be a relational structure with domain $E$, and $F$ be a finite subset of $E \backslash K(R)$. Assume furthermore that $K(R)$ is finite. Then, for every finite subset $A$ of $E$ there is some subset $A^{\prime}$ of $E \backslash F$ such that $A \approx A^{\prime}$ and $A \cap K(R)=A^{\prime} \cap K(R)$.

The proof of this lemma can obtained via the existence of a finite subset of $E$ localizing $A \cap K(R)$ (see Proposition 2.17 of [PS01]).

Trivially, we have:

Lemma 2.23. If $E^{\prime}$ is an infinite monomorphic part of $R$ then $K(R) \cap E^{\prime}=\emptyset$.

Using Lemma 2.18 and Lemma 2.22 we get: 
Lemma 2.24. If $R$ is almost-chainable then $R$ is $K(R)$-chainable.

Theorem 2.25. Let $R:=\left(E,\left(\rho_{i}\right)_{i \in I}\right)$ be a relational structure, $E^{\prime}$ be a subset of $E$ and $F:=E^{\prime} \backslash E$. Let us consider the following properties:

(i) $R$ is F-chainable;

(ii) $R$ is F-monomorphic;

(iii) $E^{\prime}$ is a monomorphic part of $R$.

Then $(i) \Rightarrow(i i) \Rightarrow($ iii $)$. If $E^{\prime}$ is infinite then $(i i) \Rightarrow(i)$. If $E^{\prime}$ is infinite and $E^{\prime}$ is a monomorphic component of $R$ then (iii) $\Rightarrow(i)$.

Proof. The implications $(i) \Rightarrow(i i) \Rightarrow($ iii $)$ are obvious. Implication $(i i) \Rightarrow(i)$ when $E^{\prime}$ is infinite is due to Fraïssé. A proof in the case of a finite signature is in [FP71]. We just recall the principle of the proof. We prove first that for each finite subset $I^{\prime}$ of $I$, each finite subset $F^{\prime}$ of $F$ and each finite subset $E^{\prime \prime}$ of $E^{\prime}$, the restriction $R^{\prime}:=R_{E^{\prime \prime} \cup F^{\prime}}^{I^{\prime}}$ is $F^{\prime}$ chainable. For that we apply Lemma 2.19. It yields an infinite subset $E_{1}^{\prime}$ of $E^{\prime}$ on which $R_{1}^{\prime}=R_{E_{1}^{\prime} \cup F^{\prime}}^{I^{\prime}}$ is $F$-chainable. Since $R$ is $F$-monomorphic, there is a local isomorphism fixing $F^{\prime}$ pointwise which send $E^{\prime \prime} \cup F^{\prime}$ into $E_{1}^{\prime} \cup F^{\prime}$; thus $R^{\prime}:=R_{\left\ulcorner E^{\prime \prime} \cup F^{\prime}\right.}^{I^{\prime}}$ is $F^{\prime}$-chainable. We conclude with Lemma 2.18. The proof of $(i i i) \Rightarrow(i)$ when $E^{\prime}$ is a component relies on the following claim:

Claim 1. Let $F^{\prime}$ be a finite subset of $F$. There is some finite subset $F^{\prime \prime}$ of $F$ containing $F^{\prime}$ such that $K\left(R_{\mid E^{\prime} \cup F^{\prime \prime}}\right)=F^{\prime \prime}$.

Proof of Claim 1. Let $x \in F$. Since the components of $R$ are the maximal monomorphic parts of $R$, the set $E^{\prime} \cup\{x\}$ is not a monomorphic part of $R$. Hence, there are $n_{x}$ and $A_{x} \not A_{x}^{\prime}$ in $[E]^{n_{x}}$ such that

$$
A_{x} \backslash\left(E^{\prime} \cup\{x\}\right)=A_{x}^{\prime} \backslash\left(E^{\prime} \cup\{x\}\right) .
$$

For each $x \in F^{\prime}$ select $A_{x}$ and $A_{x}^{\prime}$ as above. Define $V:=\bigcup_{x \in F^{\prime}} A_{x} \cup A_{x}^{\prime}$, as well as $R^{\prime}:=R_{\left\lceil E^{\prime} \cup V\right.}$, and $F^{\prime \prime}:=K\left(R^{\prime}\right)$.

Subclaim 1. $K\left(R_{\left\lceil E^{\prime} \cup F^{\prime \prime}\right.}\right)=F^{\prime \prime}$.

Proof of Subclaim 1. Since $\left(E^{\prime} \cup V\right) \backslash\left(E^{\prime} \cup F^{\prime \prime}\right)$ is finite and $F^{\prime \prime}=K\left(R^{\prime}\right)$, Lemma 2.21 asserts that $R_{\left\lceil E^{\prime} \cup V\right.}$ and $R_{\uparrow E^{\prime} \cup F^{\prime \prime}}$ have the same age and the same kernel.

Subclaim 2. $F^{\prime} \subseteq F^{\prime \prime} \subseteq F$.

Proof of Subclaim 2. Since $E^{\prime}$ is a monomorphic component of $R$, this is a monomorphic part of $R^{\prime}$ and Lemma 2.23 asserts that $K\left(R^{\prime}\right)$ is disjoint from $E^{\prime}$. This yields $F^{\prime \prime} \subseteq$ $V \backslash E^{\prime} \subseteq F$. Suppose that $F^{\prime} \nsubseteq F^{\prime \prime}$. Let $x \in F^{\prime} \backslash F^{\prime \prime}$. Then, since $F^{\prime \prime} \subseteq V \backslash E^{\prime}$, we have $F^{\prime \prime} \subseteq E \backslash\left(E^{\prime} \cup\{x\}\right)$. Thus from (7) we have $A_{x} \cap F^{\prime \prime}=A_{x}^{\prime} \cap F^{\prime \prime}$. Since $V \backslash\left(E^{\prime} \cup F^{\prime \prime}\right)$ is finite, it follows from Lemma 2.22 that there are $A, A^{\prime} \in\left[F^{\prime \prime} \cup E^{\prime}\right]^{n_{x}}$ such that $A \cap F^{\prime \prime}=A_{x} \cap F^{\prime \prime}$, $A_{x}^{\prime} \cap F^{\prime \prime}=A^{\prime} \cap F^{\prime \prime}$, and the restrictions $R_{\lceil A}^{\prime}$ and $R_{\left\lceil A^{\prime}\right.}^{\prime}$ are respectively isomorphic to $R_{\left\lceil A_{x}\right.}^{\prime}$ and $R_{\uparrow A_{x}^{\prime}}^{\prime}$. Since $E^{\prime}$ is a monomorphic component of $R^{\prime}, R_{\uparrow A}^{\prime}$ and $R_{\uparrow A^{\prime}}^{\prime}$ are isomorphic. However this implies that $A_{x} \approx A_{x}^{\prime}$, a contradiction. Therefore, $F^{\prime} \subseteq F^{\prime \prime} \subseteq V$. This completes the proof of the claim. 
With these two subclaims, the proof of Claim 1 is complete.

Now, since $E^{\prime}$ is a monomorphic part of $R_{\uparrow E^{\prime} \cup F^{\prime \prime}}$, the profile of $R_{\uparrow E^{\prime} \cup F^{\prime \prime}}$ is bounded. According to the implication $(a) \Rightarrow(c)$ in Theorem 1.5, $R_{\uparrow E^{\prime} \cup F^{\prime \prime}}$ is almost chainable. According to Lemma 2.24, $R_{\uparrow E^{\prime} \cup F^{\prime \prime}}$ is $K\left(R_{\left\lceil E^{\prime} \cup F^{\prime \prime}\right.}\right)$-chainable, that is $F^{\prime \prime}$-chainable. It follows that $R_{\uparrow E^{\prime} \cup F^{\prime}}$ is $F^{\prime}$-chainable. Since this holds for every finite subset $F^{\prime}$ of $F$, it follows from Lemma 2.18 that $R$ is $F$-chainable. Hence $(i i)$ holds. This completes the proof of Theorem 2.25 .

Theorem 1.8 follows immediately from Theorem 2.25. Indeed, if $R$ admits a finite monomorphic decomposition, we may choose one such that all the finite blocks are singletons. Hence, if $\left(E_{x}\right)_{x \in X}$ is such a decomposition, then a lexicographical sum, in any order, of the chains $\left(E_{x}, \leqslant_{x}\right)$ given by (i) of Theorem 2.25 yields a linear order on $E$ for which the $E_{x}$ are intervals and every local isomorphism preserving the $E_{x}$ 's preserves $R$.

In the special case of relational structures made of unary or binary relations, Theorem 1.8 yields the following characterization:

Corollary 2.26. If a relational structure $R$ is at most binary, it has a finite monomorphic decomposition if and only if it is a lexicographical sum of chainable relational structures indexed by a finite relational structure.

Let us say that a relational structure $R:=\left(E,\left(\rho_{i}\right)_{i \in I}\right)$ is almost multichainable if there is a finite subset $F$ of $E$ and an enumeration $\left(a_{x, y}\right)_{(x, y) \in V \times L}$ of the elements of $E \backslash F$ by a set $V \times L$, where $V$ is finite and $L$ is a linearly ordered set such that for every local isomorphism $f$ of $L$, the map $\left(1_{V}, f\right)$ extended by the identity on $F$ is a local isomorphism of $R$ (the map $\left(1_{V}, f\right)$ is defined by $\left.\left(1_{V}, f\right)(x, y):=(x, f(y))\right)$. If the map $\left(1_{V}, f\right)$ extended by the identity on $F$ is a local isomorphism of $R$ for every permutation $f$ of $L, R$ is cellular.

The notion of almost multichainability was introduced in [Pou78] and appeared in Pou81a, Pou79]. It was shown that a relational structure with polynomially bounded profile and finite kernel has the same age as an almost multichainable relational structure [Pou06]. Cellularity was introduced by J. Schmerl [Sch90] in 1990. In [Pou06] it is shown that a graph has a polynomially bounded profile if and only if it is cellular (see [Pou06]).

The following easy corollary of Theorem 1.8 show that relational structures with a finite monomorphic decomposition are essentially almost multichainable (the converse is far from be true).

Corollary 2.27. If an infinite relational structure has a finite monomorphic decomposition, then it has some restriction having the same age which is almost multichainable.

\section{Proof of Theorem 1.7}

\subsection{Preliminary steps}

From now on, we assume that $R$ has a finite monomorphic decomposition $\left(E_{x}\right)_{x \in X}$. The growth rate of the profile $\varphi_{R}$ is at most $n^{k-1}$ where $k=\left|X_{\infty}\right|$; indeed $\varphi_{R}(n)$ is bounded 
above by the number of integer vectors $\left(d_{x}\right)_{x \in X}$ such that $d_{x} \leqslant\left|E_{x}\right|$ and $\sum_{x \in X} d_{x}=n$; a more algebraic explanation is that the association $A \mapsto X^{A}$ makes the age algebra into (essentially) a subalgebra of $K\left[X_{\infty}\right]$ (see [PT12]). If the decomposition is minimal then, according to Lemma 2.15, the growth rate of the profile is at least $n^{k-1}$. We now turn to the proof that the Hilbert series is a rational fraction.

We define a total order on monomials in $\mathbb{K}[X]$ by comparing their shapes w.r.t. the degree reverse lexicographic order and breaking ties by the usual lexicographic order on monomials for some arbitrary fixed order on $X$ (recall that the reverse lexicographic order $\leqslant_{\text {revlex }}$ is defined as follow: for two integer sequences $d:=\left(d_{1}, \ldots, d_{n}\right)$ and $d^{\prime}:=$ $\left(d_{1}^{\prime}, \ldots, d_{n}^{\prime}\right)$ of same length, $d<_{\text {revlex }} d^{\prime}$ if $d \neq d^{\prime}$ and $d_{i}>d_{i}^{\prime}$ where $i$ is the largest $j \leqslant n$ such that $d_{j} \neq d_{j}^{\prime}$; for example $(4,1,1)<_{\text {revlex }}(3,3,0)$; for the degree reverse lexicographic order, one first compares the sum of the two sequences and then break ties with reverse lexicographic order; for example $\left.(3,2,0)<_{\text {degrevlex }}(4,1,1)\right)$. Beware that this total order on monomials is well founded but not a monomial order. We define the leading monomial $\operatorname{lm}(\tau)$ of an isomorphism type $\tau$ as the unique maximal monomial in the set $\left\{X^{A}: \tau(A)=\tau\right\}$.

To prove the theorem, we essentially endow the set of leading monomials with a monomial ideal structure in some appropriate polynomial ring. The point is that the Hilbert series of such monomial ideals are simple rational fractions (see e.g. [CLO97, Chapter 9, $\S 2]$; note that their presentation is in term of the Hilbert function, but this is equivalent).

Proposition 3.1. Let $\mathbb{K}\left[x_{1}, \ldots, x_{n}\right]$ be a polynomial ring whose variables have positive degree $d_{i}:=\operatorname{deg}\left(x_{i}\right)$, and let $\mathcal{I}$ be a monomial ideal. Then, the Hilbert series of $\mathcal{I}$ is of the form:

$$
\frac{P(Z)}{\left(1-Z^{d_{1}}\right) \cdots\left(1-Z^{d_{n}}\right)} .
$$

where $P \in \mathbb{Z}[Z]$.

We include the proof, as it is short and sheds some light for our purpose.

Proof. First, the Hilbert series of a principal ideal $\mathbb{K}\left[x_{1}, \ldots, x_{n}\right] . m$ generated by a monomial $m$ of degree $d$ is

$$
\frac{Z^{d}}{\left(1-Z^{d_{1}}\right) \cdots\left(1-Z^{d_{n}}\right)}
$$

Furthermore, the intersection of two principal ideals is again principal. Take now any monomial ideal $\mathcal{I}$. By Dickson Lemma, it is finitely generated by monomials $m_{1}, \ldots, m_{r}$. Therefore, the Hilbert series of $\mathcal{I}$ can be computed by inclusion-exclusion from that of the principal ideals $\left(\mathbb{K}\left[x_{1}, \ldots, x_{n}\right] . m_{i_{1}} \cap \cdots \cap \mathbb{K}\left[x_{1}, \ldots, x_{n}\right] . m_{i_{s}}\right)_{1 \leqslant i_{1}<\cdots<i_{s} \leqslant r}$.

The key property of leading monomials of age algebras is reminiscent of StanleyReisner rings. To each set $S \subseteq X$, associate the monomial $x_{S}:=\prod_{i \in S} x_{i}$. By square free factorization, any monomial $m \in \mathbb{K}[X]$ can be written in a unique way as a product $m=x_{S_{1}}^{e_{1}} \ldots x_{S_{r}}^{e_{r}}$ where $\emptyset \subset S_{1} \subset \cdots \subset S_{r} \subset X$ is a chain of non empty subsets of $X$, and the $e_{i}$ are positive. Each $S_{i}$ is a layer of $m$, and $S_{1} \subset \cdots \subset S_{r}$ is the chain support of $m$. 
Lemma 3.2. Let $m$ be a leading monomial, and $S \subseteq X$ be a layer of $m$. Then, $m x_{S}$ is again a leading monomial unless $d_{i}=\left|E_{i}\right|$ for some $i$ in $S$.

Proof. Suppose that $d_{i}<\left|E_{i}\right|$ for every $i$ in $S$. Let $A, A^{\prime}, B, B^{\prime}$ be subsets of $E$ such that $X^{A}=m, X^{B}=m x_{S}, X^{B^{\prime}}$ is the leading monomial $\operatorname{lm}(\bar{B})$, and $A^{\prime}$ is any subset of $B^{\prime}$ belonging to $\bar{A}$. Let $R_{\uparrow A}, R_{\uparrow B}, R_{\uparrow A^{\prime}}$ and $R_{\uparrow B^{\prime}}$ be the corresponding induced structures, and let $\psi$ be an isomorphism from $R_{\lceil B}$ to $R_{\uparrow B^{\prime}}$.

Setting $e:=|X|$, write respectively $\bar{d}(A)=\left(\alpha_{1}, \ldots, \alpha_{e}\right)$ and $\bar{d}(B)=\left(\beta_{1}, \ldots, \beta_{e}\right)$ the shapes of $A$ and $B$, and similarly for $A^{\prime}$ and $B^{\prime}$. Our first goal is to prove that the shapes of $B$ and $B^{\prime}$ are the same.

Claim 1. $\alpha_{p}=\beta_{p}=\beta_{p}^{\prime}=\alpha_{p}^{\prime}$ for all $p>s$ where $s:=|S|$.

Proof of Claim 1. Using that $A$ and $A^{\prime}$ have the same degree, and similarly for $B$ and $B^{\prime}$, we have:

$$
\left(\alpha_{1}, \ldots, \alpha_{e}\right) \geqslant_{\text {revlex }}\left(\alpha_{1}^{\prime}, \ldots, \alpha_{e}^{\prime}\right) \text { and }\left(\beta_{1}^{\prime}, \ldots, \beta_{e}^{\prime}\right) \geqslant_{\text {revlex }}\left(\beta_{1}, \ldots, \beta_{e}\right) .
$$

Using that $X^{B}=X^{A} x_{S}$, we get:

$$
\left(\beta_{1}, \ldots, \beta_{e}\right)=\left(\alpha_{1}+1, \ldots, \alpha_{s}+1, \alpha_{s+1}, \ldots, \alpha_{e}\right)
$$

From the inclusion $A^{\prime} \subset B^{\prime}$, we deduce that:

$$
\left(\alpha_{1}^{\prime}, \ldots, \alpha_{e}^{\prime}\right) \geqslant_{\text {revlex }}\left(\beta_{1}^{\prime}, \ldots, \beta_{e}^{\prime}\right)
$$

Altogether, using that $\leqslant_{\text {revlex }}$ is preserved on suffixes, we conclude that:

$$
\begin{aligned}
\left(\beta_{s+1}, \ldots, \beta_{e}\right) & =\left(\alpha_{s+1}, \ldots, \alpha_{e}\right) \geqslant_{\text {revlex }}\left(\alpha_{s+1}^{\prime}, \ldots, \alpha_{e}^{\prime}\right) \geqslant_{\text {revlex }}\left(\beta_{s+1}^{\prime}, \ldots, \beta_{e}^{\prime}\right) \\
& \geqslant \text { revlex }\left(\beta_{s+1}, \ldots, \beta_{e}\right),
\end{aligned}
$$

and therefore all those suffixes coincide.

Claim 2. For each $i \in S, E_{i} \cap B$ is a component of $R_{\uparrow B}$, that is a maximal monomorphic part of $R_{\uparrow B}$.

Proof of Claim 2. Suppose not. Choose $i \in S$ such that $E_{i} \cap B$ is not a component of $R_{\uparrow B}$ and is of maximal cardinality with that property. Since $\left(E_{k}\right)_{k \in X}$ is a monomorphic decomposition of $R$, then by Proposition 2.14. $\left(E_{k} \cap B\right)_{k \in X}$ is a monomorphic decomposition of $R_{\uparrow B}$. Hence, by Proposition 2.12, it refines the minimal monomorphic decomposition of $R_{\uparrow B}$. Since $E_{i} \cap B$ is not a maximal monomorphic part, there is some index $j \in X$ such that $\left(E_{i} \cup E_{j}\right) \cap B$ is still a monomorphic part of $R_{\uparrow B}$. Due to our choice of $i$, we have $\left|E_{i} \cap B\right| \geqslant\left|E_{j} \cap B\right|$. Note that this implies $\left|E_{i} \cap A\right| \geqslant\left|E_{j} \cap A\right|$. Let $a$ be the unique element of $E_{i} \cap(B \backslash A)$, pick $a^{\prime}$ in $E_{j} \cap A$, and set $A^{\prime \prime}:=A \cup\{a\} \backslash\left\{a^{\prime}\right\}$. Since the elements $a$ and $a^{\prime}$ belong to the same component of $R_{\uparrow B}, A^{\prime \prime} \approx A^{\prime \prime}$. We also have $\bar{d}(A)<_{\text {degrevlex }} \bar{d}\left(A^{\prime \prime}\right)$, a contradiction with the fact that $X^{A}$ is a leading monomial.

The proof of Claim 2 would have been simpler if the decomposition induced on $A$ by the $E_{x}$ were the components of $R_{\uparrow A}$. However this is not true in general, even with the assumption that $X^{A}$ is a leading monomial. For a simple example, take for $R$ the union 
of two non trivial cliques, and $A$ containing exactly one element in each clique; then $R_{\uparrow A}$ has a single monomorphic component and not two.

Let $S^{\prime}:=\left\{i_{1}, \ldots, i_{s}\right\}$ be $s$ distinct elements of $X$ such that $\left|E_{i_{k}} \cap B^{\prime}\right|=\beta_{k}^{\prime}$ for $k=1, \ldots, s$. Set $U:=\bigcup_{i \notin S}\left(E_{i} \cap B\right)$ and $U^{\prime}:=\bigcup_{i \notin S^{\prime}}\left(E_{i} \cap B^{\prime}\right)$.

Claim 3. $U$ is the set of all $b \in B$ such that the $B \backslash\{b\}$ contains no member of $\bar{A}$. The same statement holds for $U^{\prime}$ w.r.t. $B^{\prime}$. In particular, $\psi$ transforms $U$ into $U^{\prime}$.

Proof of Claim 3. Let $b \in U^{\prime}$. According to Claim 1, the equality $\left(\alpha_{s+1}^{\prime}, \ldots, \alpha_{e}^{\prime}\right)=$ $\left(\beta_{s+1}^{\prime}, \ldots, \beta_{e}^{\prime}\right)$ holds for any $A^{\prime} \subset B^{\prime}$ such that $A \approx A^{\prime}$. Hence, no member of $\bar{A}$ is included in $B^{\prime} \backslash\{b\}$. On the other hand, from the definition of $U, B \backslash\{b\}$ contains a member of $\bar{A}$ for every element $b \in B \backslash U$. Since $|U|=\beta_{s+1}+\cdots+\beta_{e}=\beta_{s+1}^{\prime}+\cdots+\beta_{e}^{\prime}=\left|U^{\prime}\right|$ and $\psi$ is an isomorphism from $R_{\uparrow B}$ to $R_{\uparrow B^{\prime}}$, the statement follows by cardinality count.

Claim 4. $\psi$ transforms $\left(E_{i} \cap B, i \in S\right)$ into $\left(E_{i} \cap B^{\prime}, i \in S^{\prime}\right)$.

Proof of Claim 4. Let $P(B)$ be the minimal monomorphic decomposition of $B$. By Claim 2, it is of the form $\left\{E_{i} \cap B: i \in S\right\} \cup \mathcal{P}$, where $\mathcal{P}$ is some partition of $U$. Then $\left\{\psi\left(E_{i} \cap B\right): i \in S\right\} \cup \mathcal{P}^{\prime}$, where $\mathcal{P}^{\prime}:=\{\psi(C): C \in \mathcal{P}\}$, is the minimal monomorphic decomposition $P\left(B^{\prime}\right)$ of $B^{\prime}$. Since $\left(E_{i} \cap B^{\prime}\right)_{i \in X}$ is a monomorphic decomposition of $B^{\prime}$, this is a refinement of $P\left(B^{\prime}\right)$. Using that, from Claim 3, $\psi(B \backslash U)=B^{\prime} \backslash U^{\prime}$, we obtain that $\left(E_{i} \cap B^{\prime}\right)_{i \in S^{\prime}}$ is a refinement of $\left(\psi\left(E_{i} \cap B\right)\right)_{i \in S}$. Since these two decompositions have the same cardinality, they must coincide. The statement of Claim 4 follows.

Claim 2 and Claim 4 imply immediately that $B$ and $B^{\prime}$ have the same shape. Fix now $A^{\prime}:=\psi(A)$. Using Claim $4, A^{\prime}$ is obtained from $B^{\prime}$ by removing exactly one element in each $E_{i} \cap B^{\prime}, i \in S^{\prime}$. Putting everything together, we have:

- $X^{A}$ and $X^{A^{\prime}}$ have the same shape; similarly for $B$ and $B^{\prime}$;

- $X^{B}=X^{A} x_{S}$, where $x_{S}$ is a layer of $X^{A}$; similarly $X^{B^{\prime}}=X^{A^{\prime}} x_{S^{\prime}}$;

- $X^{B} \leqslant$ lex $X^{B^{\prime}}$ and $X^{A} \geqslant_{\operatorname{lex}} X^{A^{\prime}}$.

Recall that, if two monomials have the same shape and at least one layer of size $s$, then lexicographic comparison is preserved upon changing the multiplicity of that layer (this is just applying a strictly monotone function to the exponents). Therefore, $X^{A}=X^{A^{\prime}}$ and $X^{B}=X^{B^{\prime}}$. In particular, $X^{B}=m x_{S}$ is a leading monomial, as desired.

\subsection{Final step}

Fix a chain $C:=\emptyset \subset S_{1} \subset \cdots \subset S_{r} \subseteq X$, and let $\operatorname{lm}_{C}$ be the set of leading monomials of the age algebra with this chain support. The plan is essentially to realize $\operatorname{lm}_{C}$ as the linear basis of some monomial ideal of a polynomial ring, so that the generating series of $\operatorname{lm}_{C}$ is realized as an Hilbert series. Consider the polynomial ring $\mathbb{K}\left[S_{1}, \ldots, S_{l}\right]$, with its embedding in $\mathbb{K}[X]$ by $S_{j} \mapsto x_{S_{j}}$. Let $\mathcal{I}$ be the subspace spanned by the monomials $m:=S_{1}^{r_{1}} \cdots S_{l}^{r_{l}}$ such that $d_{i}(m)>\left|E_{i}\right|$ for some $i$. It is obviously a monomial ideal. When all monomorphic blocks are infinite, $\mathcal{I}$ is the trivial ideal $\{0\}$. Consider the subspace $\mathbb{K} . \operatorname{lm}_{C}$ of $\mathbb{K}\left[S_{1}, \ldots, S_{l}\right]$ spanned by the monomials in $\operatorname{lm}_{C}$. Lemma 3.2 exactly states that 
$\mathcal{J}:=\mathbb{K} \cdot \operatorname{lm}_{C} \oplus \mathcal{I}$ is in fact also a monomial ideal of $\mathbb{K}\left[S_{1}, \ldots, S_{l}\right]$. Applying Proposition 3.1. the Hilbert series of $\mathcal{I}$ and $\mathcal{J}$ are rational fractions of the form

$$
\frac{P(Z)}{\left(1-Z^{\left|S_{1}\right|}\right) \cdots\left(1-Z^{\left|S_{l}\right|}\right)} \text {. }
$$

Hence, the same hold for $\mathcal{H}_{\mathbb{K} . \operatorname{lm}_{C}}=\mathcal{H}_{\mathcal{J}}-\mathcal{H}_{\mathcal{I}}$. Furthermore, whenever $S_{j}$ contains $i$ with $\left|E_{i}\right|<\infty$, the denominator $\left(1-Z^{\left|S_{l}\right|}\right)$ can be canceled out in $\mathcal{H}_{\mathbb{K} \text {. } \operatorname{lm}_{C}}$. The remaining denominator divides $(1-Z) \cdots\left(1-Z^{k}\right)$.

By summing up those Hilbert series $\mathcal{H}_{\mathbb{K}} \cdot \operatorname{lm}_{C}$ over all chains $C$ of subsets of $X$, we get the generating series of all the leading monomials, that is the Hilbert series of $\mathbb{K} \mathcal{A}(R)$. Hence, it is a rational fraction of the form

$$
\frac{P(Z)}{(1-Z) \cdots\left(1-Z^{k}\right)} \text {. }
$$

Recall that, if $f(z)$ is a rational fraction that is analytic at zero and has poles at points $\alpha_{1}, \ldots, \alpha_{m}$, then there exists $m$ polynomials $\left(\pi_{j}(x)\right)_{j=1}^{m}$ such that, for $n$ large enough,

$$
f_{n}=\left[z^{n}\right] f(z)=\sum_{j=1}^{m} \pi_{j}(n) \alpha_{j}^{-n}
$$

furthermore, the degree of $\pi_{j}$ is equal to the order of the pole of $f$ at $\alpha_{j}$ minus one (see e.g. [SF96, Theorem IV.9]).

Our fraction has a pole of order at most $k$ a 1 . The other poles are at roots of unity and are of order at most $k-1$. Hence $\varphi_{R}(n)$ is eventually a quasi-polynomial. Since its growth rate is bounded below by $n^{k-1}$ (Lemma 2.15) we deduce that the pole at 1 is of order $k$, that is $P(1) \neq 0$. It follows that $\varphi_{R}(n)=a n^{k-1}+O\left(n^{k-2}\right)$, for some $a \in \mathbb{R}^{+}$, as desired.

\section{Proof of Theorem 0.1}

Let $\Omega_{\mu}$ be the class of finite relational structures with signature $\mu$. The embeddability relation is a quasi order; once isomorphic structures are identified, $\Omega_{\mu}$ is a poset. Initial segments of this poset correspond to hereditary classes. If $\mathfrak{C}$ is a hereditary class, members of $\Omega_{\mu} \backslash \mathfrak{C}$ are obstructions to $\mathfrak{C}$. The minimal obstructions (minimal w.r.t. embeddability) are the bounds of $\mathfrak{C}$. Clearly $\mathfrak{C}$ is determined by its bounds. Indeed, if $\mathfrak{B}$ is a subset of $\Omega_{\mu}$, set $\uparrow \mathfrak{B}:=\left\{S \in \Omega_{\mu}: B \leqslant S\right.$ for some $\left.B \in \mathfrak{B}\right\}$ and $\operatorname{Forb}(\mathfrak{B}):=\Omega_{\mu} \backslash \uparrow \mathfrak{B}$. Then $\mathfrak{C}=\operatorname{Forb}(\mathfrak{B})$ where $\mathfrak{B}$ is the set of bounds of $\mathfrak{C}$. Hence, the fact that $\mathfrak{C}$ can be defined by a finite number of obstructions amounts to the fact that it has only finitely many bounds (considered up to isomorphy). An ideal of a poset is a non-empty and up directed initial segment. Clearly, the age of a relational structure is an ideal; the converse holds provided that $\mu$ is finite [Fra54]. By extension, the bounds of a relational structure are the bounds of its age. The decomposition of a poset, or of an initial segment thereof, into ideals is 
the backbone of the theory of ordered sets and the proof of Theorem 0.1 starts with well known properties of such decompositions.

In the sequel we suppose that $\mu$ is finite, despite that some of the results hold without this requirement. We consider two cases:

Case 1: $\mathfrak{C}$ contains no infinite antichain (w.r.t. embedability). Then, $\mathfrak{C}$ is a finite union of ages. This is a special case of a general result about posets of Erdős-Tarski [ET43]. The statement follows.

Case 2: $\mathfrak{C}$ contains an infinite antichain. Then it contains an age which cannot be defined by finitely many obstructions and contains no infinite antichain [Pou81b, 3.9 p. 329] (this fact is a special instance of a property of posets which is similar to NashWilliam's lemma on minimal bad sequences [NW63]). With this in mind, Theorem 0.1 is a consequence of the following lemma.

Lemma 4.1. An age with polynomially bounded profile can be defined by finitely many obstructions.

Lemma 4.1 is one of the many properties of relational structures with polynomially bounded profile. These properties were stated in the thesis of the first author [Pou78; some have been published in [Pou81b]. They are presented in the survey [Pou06] with a complete treatment of the case of binary structures. For the reader's convenience, the remainder of this section contains a sketch of the proof of Lemma 4.1, in two steps:

Lemma 4.2 ([ $\left.[\text { Pou06, Theorem 2.12 }]^{1}\right)$. If the profile of a relational structure $R$ is bounded by a polynomial then there is some $\vec{R}^{\prime}$ with the same age which is almost multichainable.

Lemma 4.3 ([Pou06, Theorem 4.20]). The age of an almost multichainable structure can be defined by finitely many obstructions.

Lemma 4.3 relies on the following notion and result which are are exposed in Fraïssé's book (see Chapter 13 p. 354, [Fra00]). A class $\mathfrak{C}$ of finite structures is very beautiful if for every integer $k$, the collection $\mathfrak{C}(k)$ of structures $\left(S, U_{1}, \ldots, U_{k}\right)$, where $S \in \mathfrak{C}$ and $U_{1}, \ldots, U_{k}$ are unary relations with the same domain as $S$, has no infinite antichain w.r.t. embeddability. A straightforward consequence of Higman's theorem on words (see Hig52]) is that the age of an almost multichainable structure is very beautiful. We conclude using that a very beautiful age can be defined by finitely many obstructions Pou72.

The proof of Lemma 4.2 uses the notions of kernel and of height. The height of an age $\mathcal{A}$ is an ordinal, denoted by $h(\mathcal{A})$ and defined by induction as follows: $h(\mathcal{A})=0$ if $\mathcal{A}$ has no proper sub-age; that is $\mathcal{A}$ is the age of the empty relational structure. Otherwise $h(\mathcal{A}):=\sup \left\{h\left(\mathcal{A}^{\prime}\right)+1: \mathcal{A}^{\prime}\right.$ is a proper sub-age of $\left.\mathcal{A}\right\}$. Clearly, the height is defined if and only if there is no strictly descending infinite sequence of sub-ages of $\mathcal{A}$. With this definition, it is easy to check that an age $\mathcal{A}$ has height $n$ with $n \in \mathbb{N}$ if and only if $\mathcal{A}$ is the age of a relational structure on $n$ elements. Denote by $\omega$ the first infinite ordinal, set

\footnotetext{
${ }^{1}$ Beware that it is stated there that $R$ itself is almost multichainable which might actually be an overstatement.
} 
$\omega .2:=\omega+\omega, \omega^{2}:=\omega+\omega+\cdots$. For example, we have $h(\mathcal{A})<\omega .2$ if and only if $\mathcal{A}$ is the age of an almost chainable relational structure [PS01].

Lemma 4.2 follows from the next two facts:

Fact 1 ([ Pou06, Theorem 4.30]) The profile $\varphi_{R}$ grows as a polynomial of degree $k$ if and only if $\omega \cdot(k+1) \leqslant h(\mathcal{A}(R))<\omega \cdot(k+2)$.

Fact $2\left(\left[\right.\right.$ Pou06, Theorem 4.24]) If $h(\mathcal{A})<\omega^{2}$ then $\mathcal{A}$ is the age of an almost multichainable structure.

We need two more facts before sketching the proofs of Facts 1 and 2:

Fact 3. If $\mathcal{A}$ is inexhaustible then either $\mathcal{A}$ is the age of a multichainable structure and $h(\mathcal{A})<\omega^{2}$ or $\mathcal{A}$ contains the age $\mathcal{A}_{k}$ of a multichainable structure with $h\left(\mathcal{A}_{k}\right) \geqslant \omega \cdot(k+1)$ for every integer $k$.

Fact 4 . Let $R$ be a relational structure; if every sub-age of $\mathcal{A}(R)$ is very beautiful then either $h(A) \geqslant \omega^{2}$ or $K(R)$ is finite.

Fact 3 is easy (see Proposition 4.23 of [Pou06]). Fact 4 is deeper. It is in Pou78]. It is not known if the first part of the conclusion of Fact 4 can be dropped. It can if $R$ is made of binary structures (see Theorem 4.24 of [Pou06]).

The proof of Fact 2 is by induction on $\alpha:=h(\mathcal{A})$, with $\alpha=\omega \cdot(k+1)+p$. From the induction hypothesis, every proper sub-age is the age of an almost multichainable structure; thus is very beautiful. By Fact 4, the kernel of any relational structure $R$ with age $\mathcal{A}$ is finite. Write $F:=K(R)$ and $E^{\prime}:=E \backslash K(R)$. Let $M$ be a relational structure with base $E^{\prime}$ whose local isomorphisms are those of $R_{\left\lceil E^{\prime}\right.}$ which can be extended by the identity on $F$ to local isomorphisms of $R$. Then, $M$ is age-inexhaustible and $h(\mathcal{A}(M))=\beta$ with $\beta:=\omega \cdot(k+1)$. By Fact $3, \mathcal{A}(M)$ is the age of a multichainable structure. Thus, $\mathcal{A}(R)$ is the age of an almost multichainable structure, as desired.

For the proof of Fact 1, we suppose first that $R$ is almost multichainable. As in the proof of Fact 2, we replace $R$ by $M$ and we prove that $\varphi_{M}$ is bounded by a polynomial of degree $k$ if and only if $h(\mathcal{A}(M))=\omega \cdot(k+1)$. For that, we prove first that if $h(\mathcal{A}(M))=$ $\omega .(k+1)$ then $\varphi_{M}(n) \leqslant\left(\begin{array}{c}n+k \\ k\end{array}\right)$ for every integer $n$ (Lemma 4.27 of [Pou06]). Next, in a similar way as in the proof of Theorem 2.17, we prove that $\varphi_{M}$ is bounded from below by a polynomial of degree $k$. Since $\varphi_{R}$ is bounded by a polynomial of the same degree as $\varphi_{M}$, we get the equivalence stated in Fact 1 when $R$ is almost multichainable. Now, according to Fact 2 , if $h(\mathcal{A}(R))<\omega \cdot(k+2)$ there is some $R^{\prime}$ with the same age as $R$ which is almost multichainable, and thus $\varphi_{R}=\varphi_{R^{\prime}}$ is bounded by a polynomial of degree $k$. Conversely, suppose that $\varphi_{R}$ grows as a polynomial of degree $k$. We claim that $\mathcal{A}(R)$ has an height. Indeed, otherwise $\mathcal{A}(R)$ contains an infinite antichain. Hence, as we saw in Case 2 , it contains an age $\mathcal{A}^{\prime}$ with no infinite antichain which cannot be defined by finitely many obstructions. This age has an height, say $\alpha^{\prime}$. If $\alpha^{\prime}<\omega^{2}$ then, by Fact $2, \mathcal{A}^{\prime}$ is the age of an almost multichainable structure, and thus can be defined by finitely many obstructions, which is impossible. Thus $\alpha \geqslant \omega^{2}$. But then by Fact $3, \mathcal{A}^{\prime}$ contains ages of height $\omega \cdot\left(k^{\prime}+1\right)$ for every $k^{\prime}$ and thus $\varphi_{R}$ grows faster than every polynomial. A contradiction. This proves our claim. Now, let $\alpha=h(\mathcal{A}(R))$. By the same token, $\alpha<\omega^{2}$, hence $\mathcal{A}(R)$ is the age of an almost multichainable structure. And thus $\omega \cdot(k+1) \leqslant \alpha<\omega \cdot(k+2)$, as desired. 


\section{A Examples of relational structures and age algebras}

\section{A.1 Examples coming from graphs and digraphs}

A graph $G:=(V, \mathcal{E})$ being considered as a binary irreflexive and symmetric relation, its profile $\varphi_{G}$ is the function which counts, for each integer $n$, the number $\varphi_{G}(n)$ of induced subgraphs on $n$ elements subsets of $V(G)$, isomorphic subgraphs counting for one. Graphs with profile bounded by a polynomial have been described in [Pou06, BBSS09]. According to [BBSS09] the profile is either a quasi-polynomial or is eventually bounded below by the partition function $\wp$, where $\wp(n)$ is the number of integer partitions of $n$. Tournaments with polynomially bounded profile have been characterized in [BP10] as lexicographical sums of acyclic tournaments indexed by a finite tournament. They admit finite monomorphic decomposition, hence their profile is a quasi-polynomial.

Example A.1. Trivially, if $G$ is an infinite clique $K_{\infty}$ or coclique $\bar{K}_{\infty}$, then there is a single isomorphic type for each $d$, hence $\varphi_{G}(n)=1$. There is one monomorphic component $E_{x}=E$, the age algebra is $\mathbb{K}[x]$, and its Hilbert series is $\frac{1}{1-Z}$. If instead one consider digraphs, we recover the same age and age algebra from any infinite chain $\mathbb{N}, \mathbb{Z}, \mathbb{Q}, \mathbb{R}$, etc. or antichain.

A bit less trivial is the fact that $\varphi_{G}$ is bounded if and only if $G$ is almost constant in the sense of Fraïssé [Fra00] (there exists a finite subset $F_{G}$ of vertices such that two pairs of vertices having the same intersection on $F_{G}$ are both edges or both non-edges).

Example A.2. Let $G$ be the direct sum $K_{\omega} \oplus \cdots \oplus K_{\omega}$ of $k$ infinite cliques (or chains) $E_{1}, \ldots, E_{k}$ (see figure in Table 1). The $E_{i}$ form the monomorphic components. The profile counts the number $\varphi_{G}(n)=p_{k}(n) \simeq \frac{n^{k}}{(k+1) ! k !}$ of integer partitions with at most $k$ parts. The age algebra is the ring of symmetric polynomials $\operatorname{Sym}(X)$ on $k$ variables whose Hilbert series is $\frac{1}{(1-Z) \cdots\left(1-Z^{k}\right)}$.

Examples A.3. Let $G$ be the direct sum $K_{\omega} \oplus \bar{K}_{\omega}$ of an infinite clique and an infinite independent set. Then, $\varphi_{G}(n)=n$ for $n \geqslant 1$, and $\mathcal{H}_{G}=1+\frac{Z}{(1-Z)^{2}}=\frac{1-Z+Z^{2}}{(1-Z)^{2}}=\frac{1+Z^{3}}{(1-Z)\left(1-Z^{2}\right)}$. Hence, the Hilbert series has one representation as a rational fraction with a numerator with some negative coefficient, and another with all coefficients non-negative.

This Hilbert series coincides further with that of Examples A.4, and A.18 for $k=$ 2. Still, in the first and third case, there are two infinite monomorphic components whereas in the second there are three: one finite and two infinite. Furthermore, the age algebra is finitely generated and even Cohen-Macaulay in the first (take the free subalgebra generated by a point and a 2-chain, and take as module generators the empty set and a 3 -chain) and third case, but not in the second.

Example A.4. Let $G$ be the direct sum $K_{(1, \omega)} \oplus \bar{K}_{\omega}$ of an infinite wheel and an infinite independent set. There are two infinite monomorphic components, $E_{1}$ the set of leaves of the wheel and $E_{2}$ the independent set, and one finite, $E_{3}$, containing the center $c$ of the wheel. Each isomorphism type consists of a wheel and an independent set, so the Hilbert series is $\mathcal{H}_{G}(Z)=\left(1+\frac{Z^{2}}{1-Z}\right) \frac{1}{1-Z}=\frac{1-Z+Z^{2}}{(1-Z)^{2}}=\frac{1+Z^{3}}{(1-Z)\left(1-Z^{2}\right)}$. 
What makes this relational structure special is that the monomorphic decomposition $\left(E_{1}, E_{2}, E_{3}\right)$ is minimal, whereas $\left(E_{1}, E_{2}\right)$ is not a minimal monomorphic decomposition of the restriction of $R$ to $E_{1} \cup E_{2}$. We now prove that this causes the age algebra not to be finitely generated. Consider the subalgebra $\mathcal{B}:=\mathbb{K}\left[e_{1}(E)\right]$. In each degree $d$, it is spanned by the sum $b_{d}$ of all subsets of size $d$ of $E$. Key fact: any element $s$ of $\mathbb{K} \mathcal{A}(R)$ can be uniquely written as $s=: a(s)+b(s)$ where $b(s)$ is in $\mathcal{B}$, and all subsets in the support of $a(s)$ contain the unique element $c$ of $E_{3}$. Note in particular that $a(s)^{2}=0$ for any $s$ homogeneous of positive degree. Let $S$ be a finite generating set of the age algebra made of homogeneous elements of positive degree. By the remark above, $\{a(s), s \in S\}$ generates $\mathbb{K} \mathcal{A}(R)$ as a $\mathcal{B}$-module. It follows that the graded dimension of $\mathbb{K} \mathcal{A}(R)$ is bounded by $|S|$, a contradiction.

Example A.5. When extending Example A.2 to infinitely many cliques the age algebra becomes the ring Sym of symmetric functions whose Hilbert series is $\mathcal{H}_{R}(Z)=\prod_{d \geqslant 1} \frac{1}{\left(1-Z^{d}\right)}$.

Example A.6. If $G$ is an infinite path, then the finite restrictions are direct sums of paths. Therefore, the profile counts the number of integer partitions of $n$. The age algebra is the free commutative algebra generated by the paths of length $1,2, \ldots$, which is again isomorphic to Sym. However, this time, the monomorphic components are reduced to singletons.

Example A.7. If $G$ is the Rado graph, then $\varphi_{G}(n)$ counts the total number of unlabelled graphs. The age algebra is the free commutative algebra generated by the connected graphs. Its Hilbert series is $\mathcal{H}_{R}(Z)=\prod_{d \geqslant 1} \frac{1}{\left(1-Z^{d}\right)^{c} d}$, where $c_{d}$ is the number of connected graphs with $d$ vertices.

Example A.8. Let $G$ be the lexicographic sum tournament obtained by substituting each point $i$ of the cycle $C_{3}:=\{(1,2),(2,3),(3,1)\}$ on $\{1,2,3\}$ by the chain $\mathbb{N}$. The three chains $\left(E_{1}, E_{2}, E_{3}\right)$ give the minimal monomorphic decomposition of $G$, but this decomposition is not recursively minimal and the age algebra is not finitely generated because, as above, $\left(E_{1}, E_{2}\right)$ is not the minimal monomorphic decomposition of $R$ restricted to $E_{1} \cup E_{2}$ (see [PT12]).

Example A.9. Take the direct sum $\bar{K}_{\infty} \prec G$ of infinitely many copies of a finite connected graph $G$. The age algebra is the free commutative algebra generated by the (finitely many) connected induced subgraphs of $G$. Taking for $G$ the graph $K_{1,1}$, one gets the infinite matching. The age algebra is finitely generated, whereas there are infinitely many monomorphic blocks. The extension of this example to $G$ a finite relational structure is straightforward.

Examples A.10. Let $G$ be the simple graph consisting of the direct sum of an infinite wheel and an infinite matching. Each isomorphism type is the direct sum of a wheel, an independent set, and a matching. Therefore, the Hilbert series is $\mathcal{H}_{G}(Z)=\frac{Z}{1-Z} \frac{1}{1-Z} \frac{1}{1-Z^{2}}$, and the profile has polynomial growth: $\varphi_{G}(n) \sim a n^{2}$. There is one infinite monomorphic block (the leaves of the wheel), and infinitely many finite ones (the center of the wheel, and the edges of the matching). The age algebra is not finitely generated because $G$ contains as restriction the graph of Example A.4 whose age algebra is not finitely generated. 


\section{A.2 Examples coming from groups}

We first look at orbital profiles. The fact that they are special cases of profiles is easy to prove. In fact, for every permutation group $G$ on a set $E$, there is a relational structure $R$ on $E$ such that Aut $R=\bar{G}$ (the topological closure of $G$ in the symmetric group $\mathfrak{G}(E)$, equipped with the topology induced by the product topology on $E^{E}, E$ being equipped with the discrete topology). In particular, $\theta_{G}(n)=\varphi_{R}(n)$ for all $n$. Oligomorphic groups are quite common. Indeed, if $E$ is denumerable, then $G$ is oligomorphic if and only if the complete theory of $R$ is $\aleph_{0}$-categorical [RN59]. Cameron conjectured that the orbital profile $\theta_{G}$ is polynomial (in the sense that $\theta_{G}(n) \sim a n^{k}$ ) provided that it is bounded by some polynomial. This particular consequence of Conjecture 1.3 has not been solved yet.

Example A.11. Let $G$ be the trivial group on an $m$ element set $E$. Set $R:=\left(E, u_{1}, \ldots, u_{m}\right)$, where each $u_{i}$ is a unary relation defining the $i$-th element of $E$. Then, $\theta_{G}(n)=\varphi_{R}(n)=$ $\left(\begin{array}{c}m \\ n\end{array}\right)$.

Example A.12. Let $G$ be the symmetric group $\mathfrak{S}_{N}$, acting on the set of all pairs $\{i, j\}$ of $\{1, \ldots, N\}$ by $\sigma(\{i, j\})=\{\sigma(i), \sigma(j)\}$. Then, the orbits of $G$ are the unlabelled graphs on $N$ vertices, counted by number of edges. In the age algebra, the product of two graphs is the sum (with multiplicities) of all graphs that can be obtained by superposing them without overlapping edges.

Example A.13. Let $\mathcal{A}$ be a finite alphabet with $k$ elements, and let $\mathcal{A}^{*}$ be the set of words over $\mathcal{A}$. Then each word can be viewed as a finite chain coloured by $k$ colors. Hence $\mathcal{A}^{*}$ is the age of the relational structure $R$ made of the chain $\mathbb{Q}$ of rational numbers divided into $k$ colors in such a way that, between two distinct rational numbers, all colors appear. Furthermore, $R$ is homogeneous in the sense that every local isomorphism of $R$ with finite domain extends to an automorphism of $R$, hence the set of orbits of $G:=\operatorname{Aut}(R)$ can be identified to $\mathcal{A}(R)$. As pointed out by Cameron [Cam97, the age algebra $\mathbb{K} \mathcal{A}(R)$ is isomorphic to the shuffle algebra over $\mathcal{A}$, an important object in algebraic combinatorics (see [Lot97]). A more sophisticated example of shuffle algebra is presented in Subsection A.4.

Example A.14. Let $G:=$ Aut $\mathbb{Q}$, where $\mathbb{Q}=(\mathbb{Q}, \leqslant)$ is the chain of rational numbers. Then, $\theta_{G}(n)=\varphi_{\mathbb{Q}}(n)=1$ for all $n$. There is a single monomorphic block, and $\mathbb{K} \mathcal{A}(R) \approx \mathbb{K}[x]$.

Example A.15. Let $R:=\left(\mathbb{Q}, \leqslant, u_{1}, \ldots, u_{k}\right)$, where $\mathbb{Q}$ is the chain of rational numbers, and $u_{1}, \ldots, u_{k}$ are $k$ unary relations which divide $Q$ into $k$ non-trivial intervals $E_{1}, \ldots, E_{k}$. Then, $\varphi_{R}(n)=\left(\begin{array}{c}n+k-1 \\ k-1\end{array}\right)$ and $\mathcal{H}_{R}=\frac{1}{(1-Z)^{k}}$. The $E_{i}$ 's are the monomorphic blocks and $\mathbb{K} \mathcal{A}(R) \approx \mathbb{K}[X]$.

Example A.16. Let $G^{\prime}$ be the wreath product $G^{\prime}:=G 2 \mathfrak{S}_{\mathbb{N}}$ of a permutation group $G$ acting on $\{1, \ldots, k\}$ and of $\mathfrak{S}_{\mathbb{N}}$, the symmetric group on $\mathbb{N}$. Looking at $G^{\prime}$ as a permutation group acting on $E^{\prime}:=\{1, \ldots, k\} \times \mathbb{N}$, then $G^{\prime}=$ Aut $R^{\prime}$ for some relational structure $R^{\prime}$ on $E^{\prime}$; moreover, for all $n, \theta_{G^{\prime}}(n)=\varphi_{R^{\prime}}(n)$. Among the possible $R^{\prime}$ take $R \imath \mathbb{N}:=\left(E^{\prime}, \equiv,\left(\bar{\rho}_{i}\right)_{i \in I}\right)$, where $\equiv$ is $\left\{((i, n),(j, m)) \in E^{\prime 2}: i=j\right\}, \bar{\rho}_{i}:=\left\{\left(\left(x_{1}, m_{1}\right), \ldots,\left(x_{n_{i}}, m_{n_{i}}\right)\right):\left(x_{1}, \ldots, x_{n_{i}}\right) \in\right.$ $\left.\rho_{i},\left(m_{1}, \ldots, m_{n_{i}}\right) \in \mathbb{N}^{n_{i}}\right\}$, and $R:=\left(\{1, \ldots, k\},\left(\rho_{i}\right)_{i \in I}\right)$ is a relational structure having 
signature $\mu:=\left(n_{i}\right)_{i \in I}$ such that $\operatorname{Aut} R=G$. The relational structure $R \imath \mathbb{N}$ decomposes into $k$ monomorphic blocks, namely the equivalence classes of $\equiv$.

As it turns out Cam90, $\mathcal{H}_{R \backslash \mathbb{N}}$ is the Hilbert series of the invariant ring $\mathbb{K}[X]^{G}$ of $G$, that is the subring of the polynomials in $X$ which are invariant under the action of $G$. In fact, the identification of the age algebra as a subring of $\mathbb{K}[X]$ gives an isomorphism with $\mathbb{K}[X]^{G}$. As it is well known, this ring is Cohen-Macaulay, and the Hilbert series is a rational fraction of the form given in Theorem 1.7 , where the coefficients of $P(Z)$ are non-negative.

When $G$ is the trivial group, one recovers the polynomial ring $\mathbb{K}[X]$, as in Example A.15.

Problem A.17. Find an example of a permutation group $G^{\prime}$ with no finite orbit, such that the orbital profile of $G^{\prime}$ has polynomial growth, but the generating series is not the Hilbert series of the invariant ring $\mathbb{K}[X]^{G}$ of some permutation group $G$ acting on a finite set $X$.

\section{A.3 Examples coming from permutation groupoids}

Let $X$ be a set. A local bijection of $X$ is a bijective function $f$ whose domain $\operatorname{dom} f$ and image $\operatorname{im} f$ are subsets of $X$. The inverse $f^{-1}$ of a local bijection $f$, its restriction $f_{\left\lceil X^{\prime}\right.}$ to a subset $X^{\prime}$ of $\operatorname{dom} f$ (with codomain $f\left(X^{\prime}\right)$ ), and the composition $f \circ g$ of two local bijections $f$ and $g$ such that $\operatorname{im} g=\operatorname{dom} f$ are defined in the natural way. A set $G$ of local bijections of $X$ is called a permutation groupoid if it contains the identity and is stable by restriction, inverse, and composition. It is furthermore locally closed if a local bijection $f$ is in $G$ whenever all its finite restrictions are. Obviously, the closure $\downarrow G$ of a permutation group $G$ by restriction is a permutation groupoid. More interestingly, the local isomorphisms of a relational structure form a locally closed permutation groupoid, and reciprocally, any locally closed permutation groupoid $G$ can be obtained from a suitable relational structure $R_{G}$ on $X$.

The wreath product construction of an age algebra matching the invariant ring $\mathbb{K}[X]^{G}$ of a permutation group $G$ (see Example A.16) can be extended straightforwardly to permutation groupoids. Many, but not all, properties of invariant rings of permutation groups carry over (see Table 1 and [PT05, PT12]); in particular, the invariant ring is still a module over symmetric functions, but not necessarily Cohen-Macaulay.

Examples A.18. Take $n \in \mathbb{N} \cup\{\infty\}$ and let $G$ be the permutation groupoid of the strictly increasing local bijections of $\{1, \ldots, n\}$, or equivalently of the local isomorphisms of the chain $1<\cdots<n$. Then, $\mathbb{K}[X]^{G}$ is the ring $\operatorname{QSym}(X)$ of quasi-symmetric polynomials on the ordered alphabet $X$, as introduced by I. Gessel [Ges84]. As shown by F. Bergeron and C. Reutenauer, $\mathcal{H}_{\mathrm{QSym}(X)}=\frac{P_{n}(Z)}{(1-Z)\left(1-Z^{2}\right) \cdots\left(1-Z^{n}\right)}$, where the coefficients of $P_{n}(Z)$ are non negative. In fact, the ring is Cohen-Macalay GW03.

Taking the same groupoid $G$, and letting it act naturally on respectively pairs, couples, $k$-subsets, or $k$-tuples of elements of $\{1, \ldots, n\}$, yield respectively the (un)oriented (hyper)graph quasi-symmetric polynomials of [NTT04]. 
The $r$-quasi symmetric polynomials [Hiv05] can be realized as well as the age algebra of a relational structure (but not as the invariant ring of a permutation groupoid). Namely start from the relational structure of Example A.2, and add another $2 r$-ary relation $\rho$ such that $\rho\left(x_{1}, \ldots, x_{r}, y_{1}, \ldots, y_{r}\right)$ holds if $x_{1}, \ldots, x_{r}$ are distinct and in some block $E_{i}$ and $y_{1}, \ldots, y_{r}$ are distinct and in some block $E_{j}$ with $i<j$. For $r=1$, one recovers the relational structure giving quasi symmetric functions and for $r=0$ the relational structure giving symmetric polynomials.

Example A.19. Let $G$ be the permutation groupoid on $\{1,2,3\}$ generated by the local bijection $1 \mapsto 2$. Then, $G$ is the restriction of the finite permutation group $\langle(1,2),(3,4)\rangle$ whose invariant ring is Cohen-Macaulay. However, the age algebra $\mathbb{K}[X]^{G}$ itself is not Cohen-Macaulay. In fact, the numerator of the Hilbert series cannot be chosen with non-

negative coefficients. Indeed, $\mathcal{H}_{\mathbb{K}[X]^{G}}=\frac{1-Z+2 Z^{2}-Z^{3}}{(1-Z)^{3}}$, and the coefficient of highest degree in the product of the numerator by $\frac{\left(1-Z^{n_{1}}\right)\left(1-Z^{n_{2}}\right)\left(1-Z^{n_{3}}\right)}{(1-Z)^{3}}$ is always -1 .

\section{A.4 Example: the shuffle algebra of planar tree polynomials}

As a final example, and in order to illustrate the limits of monomorphic decompositions, we consider the shuffle algebra of planar tree polynomials $\left(\mathbb{K}\{x\}_{\infty}\right.$, $)$ ) which arises naturally in the study of non associative analogues of the exponential and logarithm [DG04, Ger05]. We realize $\left(\mathbb{K}\{x\}_{\infty}, \amalg\right)$ as an age algebra, and show that the minimal monomorphic decomposition of the underlying relational structure is trivial, and in particular infinite.

In this section, all trees are rooted, ordered, and unlabelled (in the papers cited above, those trees are called planar). A tree is reduced if all its internal nodes are of arity at least two. Let $\mathcal{T}_{i}$ be the set of all reduced trees with $i$ leaves and set $\mathcal{T}:=\bigcup_{d=0}^{\infty} \mathcal{T}_{d}$. By convention, $\mathcal{T}_{0}$ contains the empty reduced tree with zero leaves. Denoting leaves by "o" and subtrees using parentheses, one has:

$$
\mathcal{T}_{1}=\{\circ\}, \quad \mathcal{T}_{2}=\{(\circ, \circ)\}, \quad \mathcal{T}_{3}=\{(\circ,(\circ, \circ)),(\circ, \circ, \circ),((\circ, \circ), \circ)\} .
$$

Those trees are counted by the sequence of Schröder numbers or super Catalan numbers (\#A001003 of [FI12]):

$$
1,1,1,3,11,45,197,903,4279,20793,103049, \ldots
$$

To each tree $\tau$ can be associated a canonical reduced tree $\operatorname{red}(\tau)$ by contracting all paths in $\tau$ to suppress intermediate nodes of arity 1 . Given a subset $A$ of the leaves of $\tau$, one defines the contraction of $\tau$ on $A$ as $\tau_{\uparrow A}:=\operatorname{red}\left(\tau^{\prime}\right)$, where $\tau^{\prime}$ is the subtree induced by $\tau$ on the set of all nodes of $\tau$ between the root and the leaves in $A$.

Lemma A.20. Let $\tau$ and $\tau^{\prime}$ be two reduced trees with $d$ leaves. Then, $\tau=\tau^{\prime}$ if and only if the contractions $\tau_{\uparrow A}$ and $\tau_{\uparrow A}^{\prime}$ are identical for any 3-subset $A$ of the leaves.

Proof. The "only if" statement is obvious, and we turn to the "if" statement. For simplicity we denote the leaves $\{1, \ldots, d\}$. Consider an internal node of $\tau$; since $\tau$ is reduced, this 
node is uniquely caracterized by the interval $[i, j]$ formed by the leaves under it. We call $[i, j]$ a node interval. Note that a reduced tree is uniquely caracterized by the collection of its node invervals. Note further that an interval $[i, j]$ is a node interval if and only if:

- for $k<i$, the restriction $\tau_{\lceil\{k, i, j\}}$ is the tree $(\circ,(\circ, \circ))$, and

- for $k>j$, the restriction $\tau_{\{\{i, j, k\}}$ is the tree $((\circ, \circ), \circ)$.

This concludes the proof.

We now construct a relational structure whose isomorphism types are given by the reduced trees. Consider the infinite rooted ordered tree $T$ such that each internal node has infinitely many children, each alternatively a leaf or a copy of $T$ (see Figure 1). Let $E$ be the set of leaves of $T$. To each finite subset $A$ of $E$, we associate the reduced tree $T_{\lceil A}$ obtained by contracting $T$ on $A$ as in the finite case (see the example in Figure 1 ).
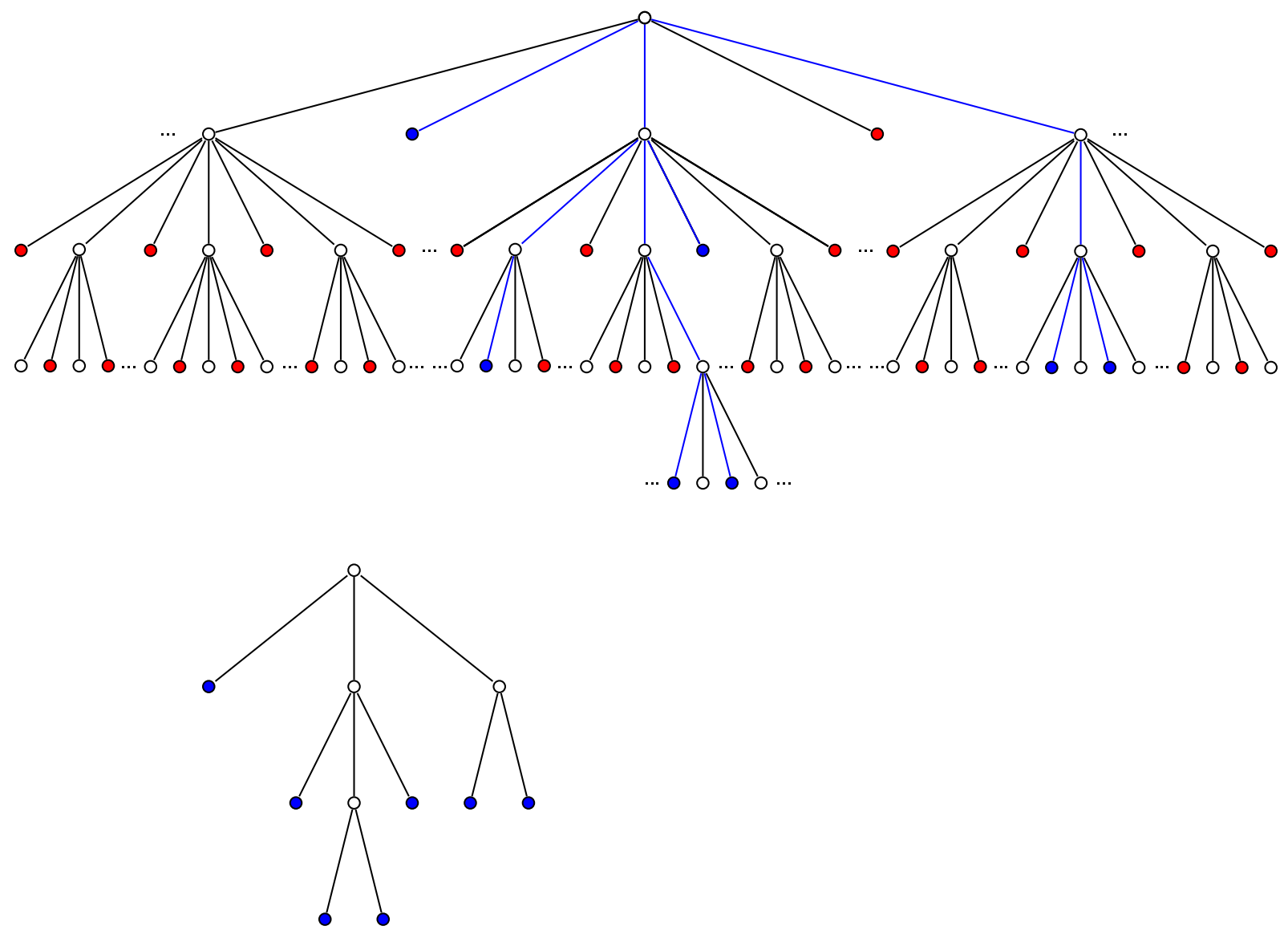

Figure 1: Above: the infinite tree $T$; in white, its internal nodes; in red and blue: its leaves. Below: the reduced tree $T_{\uparrow A}$, where $A$ is the set of blue leaves in $T$.

Let $R$ be the relational structure obtained by endowing $E$ with:

- the total order $<$ induced by left-right infix order on $T$; 
- for each of the three reduced trees $\tau$ in $\mathcal{T}_{3}$, the ternary relation

$$
\rho_{\tau}:=\left\{(x, y, z): x<y<z \text { and } T_{\{\{x, y, z\}}=\tau\right\} .
$$

Note that the relational structure $(E,<)$ is isomorphic to the chain of rationals.

Proposition A.21. The profile of $R$ counts the reduced trees. Namely, $A \approx A^{\prime}$ if and only if $T_{\lceil A}=T_{\left\lceil A^{\prime}\right.}$, and any reduced tree $\tau \in \mathcal{T}$ arises this way.

Proof. We need only to consider the case where $A$ and $A^{\prime}$ are of the same size $d$. Write $A=\left\{x_{1}, \ldots, x_{d}\right\}$ and $A^{\prime}=\left\{x_{1}^{\prime}, \ldots, x_{d}^{\prime}\right\}$ along the total order $<$. Take $\{i, j, k\}$ a 3 -subset of leaves of $T_{\uparrow A}$ (and of $T_{\left\lceil A^{\prime}\right.}$ ). Thanks to the compatibility of contraction with <, one has

$\left(T_{\lceil A}\right)_{\{\{i, j, k\}}=T_{\left\{\left\{x_{i}, x_{j}, x_{k}\right\}\right.}$ and similarly for $A^{\prime}$. Therefore $A$ and $A^{\prime}$ are isomorphic if and only if $T_{\lceil A}$ and $T_{\left\lceil A^{\prime}\right.}$ have the same 3-leaf contractions. We conclude by reconstruction using Lemma A.20.

For the last statement, choose any of the straightforward embeddings of $\tau$ in $T$.

Proposition A.22. The minimal monomorphic decomposition of $R$ is trivial: each of its monomorphic component is a singleton.

Proof. Since a subset of a monomorphic part is a monomorphic part (see Lemma 2.11), it is sufficient to prove that there is no two-element monomorphic part. Take $a<b$ in $E$. In between $a$ and $b$ in $T$ there is a full-blown copy of $T$. Thus, we can take two leaves $c, d$ of $T$ with $a<c<d<b$ such that $c, d$ have a common ancestor which is neither an ancestor of $a$ nor of $b$. Then,

$$
T_{\{\{a, c, d\}}=(\circ,(\circ, \circ)) \neq((\circ, \circ), \circ)=T_{\{\{b, c, d\}} .
$$

Therefore $\{a, b\}$ is not a monomorphic part.

Proposition A.23. The age algebra of $R$ is isomorphic to the shuffle algebra of planar tree polynomials $\left(\mathbb{K}\{x\}_{\infty}, ш\right)$.

Proof. Let $\tau, \tau_{1}, \tau_{2}$ be three reduced trees. The structure coefficient $c_{\tau_{1}, \tau_{2}}^{\tau}$ is obtained by taking any $A$ such that $\tau=T_{\lceil A}$ and counting the number of $A_{1} \uplus A_{2}=A$ such that $\tau_{1}=T_{\left\lceil A_{1}\right.}$ and $\tau_{2}=T_{\left\lceil A_{2}\right.}$. This matches with the definition of the structure constants of the shuffle product on planar tree polynomials (see [Ger05, Section 3]).

\section{References}

[AA05] M. H. Albert and M. D. Atkinson. Simple permutations and pattern restricted permutations. Discrete Math., 300(1-3):1-15, 2005.

[AAB07] M. H. Albert, M. D. Atkinson, and Robert Brignall. Permutation classes of polynomial growth. Ann. Comb., 11(3-4):249-264, 2007. 
[BBM07] József Balogh, Béla Bollobás, and Robert Morris. Hereditary properties of combinatorial structures: posets and oriented graphs. J. Graph Theory, 56(4):311332, 2007.

[BBSS09] József Balogh, Béla Bollobás, Michael Saks, and Vera T. Sós. The unlabelled speed of a hereditary graph property. J. Combin. Theory Ser. B, 99(1):9-19, 2009 .

[BHV08] Robert Brignall, Sophie Huczynska, and Vincent Vatter. Decomposing simple permutations, with enumerative consequences. Combinatorica, 28(4):385-400, 2008.

[Bol98] Béla Bollobás. Hereditary properties of graphs: asymptotic enumeration, global structure, and colouring. In Proceedings of the International Congress of Mathematicians, Vol. III (Berlin, 1998), number Extra Vol. III, pages 333-342 (electronic), 1998.

[BP10] Youssef Boudabbous and Maurice Pouzet. The morphology of infinite tournaments; application to the growth of their profile. European J. Combin., 31(2):461-481, 2010.

[Cam90] Peter J. Cameron. Oligomorphic permutation groups, volume 152 of London Mathematical Society Lecture Note Series. Cambridge University Press, Cambridge, 1990.

[Cam97] Peter J. Cameron. The algebra of an age. In Model theory of groups and automorphism groups (Blaubeuren, 1995), volume 244 of London Math. Soc. Lecture Note Ser., pages 126-133. Cambridge Univ. Press, Cambridge, 1997.

[Cam09] Peter Cameron. Oligomorphic permutation groups. In Mohan Delampady N. S. Narasimha Sastry, T. S. S. R. K. Rao and B. Rajeev, editors, Perspectives in Mathematical Sciences II: Pure Mathematics, pages 37-61. World Scientific, Singapore, 2009.

[CLO97] David Cox, John Little, and Donal O'Shea. Ideals, varieties, and algorithms. Springer-Verlag, New York, second edition, 1997. An introduction to computational algebraic geometry and commutative algebra.

[DG04] Vesselin Drensky and Lothar Gerritzen. Nonassociative exponential and logarithm. J. Algebra, 272(1):311-320, 2004.

[ET43] P. Erdős and A. Tarski. On families of mutually exclusive sets. Ann. of Math. (2), 44:315-329, 1943.

[FI12] OEIS Foundation Inc. The on-line encyclopedia of integer sequences, 2012.

[FP71] Roland Fraïssé and Maurice Pouzet. Interprétabilité d'une relation pour une chaîne. C. R. Acad. Sci. Paris Sér. A-B, 272:A1624-A1627, 1971.

[Fra54] Roland Fraïssé. Sur quelques classifications des systèmes de relations. Publ. Sci. Univ. Alger. Sér. A., 1:35-182 (1955), 1954. 
[Fra65] Claude Frasnay. Quelques problèmes combinatoires concernant les ordres totaux et les relations monomorphes. Ann. Inst. Fourier (Grenoble), 15(fasc. 2):415-524, 1965 .

[Fra71] Roland Fraïssé. Cours de logique mathématique. Tome 1: Relation et formule logique. Gauthier-Villars Éditeur, Paris, 1971. Deuxième édition revue et modifiée, Collection de "Logique Mathématique", Série A, No. 23.

[Fra00] Roland Fraïssé. Theory of relations, volume 145 of Studies in Logic and the Foundations of Mathematics. North-Holland Publishing Co., Amsterdam, revised edition, 2000. With an appendix by Norbert Sauer.

[Ger05] L. Gerritzen. Planar shuffle product, co-addition and the non-associative exponential. 2005. arXiv:math/0502378v1 [math.RA].

[Ges84] Ira M. Gessel. Multipartite $P$-partitions and inner products of skew Schur functions. In Combinatorics and algebra (Boulder, Colo., 1983), volume 34 of Contemp. Math., pages 289-317. Amer. Math. Soc., Providence, RI, 1984.

[GW03] A. M. Garsia and N. Wallach. Qsym over Sym is free. J. Combin. Theory Ser. A, 104(2):217-263, 2003.

[Hig52] Graham Higman. Ordering by divisibility in abstract algebras. Proc. London Math. Soc. (3), 2:326-336, 1952.

[Hiv05] Florent Hivert. Local actions of the symmetric group and generalisations of quasi-symmetric functions, 2005.

[KK03] Tomáš Kaiser and Martin Klazar. On growth rates of closed permutation classes. Electron. J. Combin., 9(2):Research paper 10, 20 pp. (electronic), 2002/03. Permutation patterns (Otago, 2003).

[Kla08a] M. Klazar. Overview of some general results in combinatorial enumeration. Arxiv preprint arXiv:0803.4292, 2008.

[Kla08b] Martin Klazar. On growth rates of permutations, set partitions, ordered graphs and other objects. Electron. J. Combin., 15(1):Research Paper 75, 22, 2008.

[Lot97] M. Lothaire. Combinatorics on words. Cambridge Mathematical Library. Cambridge University Press, Cambridge, 1997. With a foreword by Roger Lyndon and a preface by Dominique Perrin, Corrected reprint of the 1983 original, with a new preface by Perrin.

[MT04] Adam Marcus and Gábor Tardos. Excluded permutation matrices and the Stanley-Wilf conjecture. J. Combin. Theory Ser. A, 107(1):153-160, 2004.

[NTT04] Jean-Christophe Novelli, Jean-Yves Thibon, and Nicolas M. Thiéry. Algèbres de Hopf de graphes. C. R. Math. Acad. Sci. Paris, 339(9):607-610, 2004. doi:10.1016/j.crma.2004.09.012, arXiv:0812.3407v1 [math.CO].

[NW63] C. St. J. A. Nash-Williams. On well-quasi-ordering finite trees. Proc. Cambridge Philos. Soc., 59:833-835, 1963.

[OP11] Djamila Oudrar and Maurice Pouzet. Profile and hereditary classes of relational structures. In H.Ait Haddadene, I.Bouchemakh, M.Boudar, and S.Bouroubi 
(LAID3), editors, Proceedings ISOR'11, International Symposium on Operational Research, Algiers, Algeria, May 30-June 2, 2011.

[Pou72] Maurice Pouzet. Un bel ordre d'abritement et ses rapports avec les bornes d'une multirelation. C. R. Acad. Sci. Paris Sér. A-B, 274:A1677-A1680, 1972.

[Pou76] Maurice Pouzet. Application d'une propriété combinatoire des parties d'un ensemble aux groupes et aux relations. Math. Z., 150(2):117-134, 1976.

[Pou78] Maurice Pouzet. Sur la théorie des relations. Thèse d'état, Université ClaudeBernard, Lyon 1, 1978.

[Pou79] Maurice Pouzet. Relation minimale pour son âge. Z. Math. Logik Grundlag. Math., 25(4):315-344, 1979.

[Pou80] Maurice Pouzet. The asymptotic behavior of a class of counting functions. In M. Deza and I. G. Rosenberg, editors, Combinatorics 79. Part II, Proceedings of the Colloquium held at the University of Montréal, Montreal, Que., June 11-16, 1979, volume 9, pages 223-224. Elsevier, 1980.

[Pou81a] M. Pouzet. Relations impartibles. Dissertationes Math. (Rozprawy Mat.), 193:43, 1981.

[Pou81b] Maurice Pouzet. Application de la notion de relation presque-enchaînable au dénombrement des restrictions finies d'une relation. Z. Math. Logik Grundlag. Math., 27(4):289-332, 1981.

[Pou06] Maurice Pouzet. The profile of relations. Glob. J. Pure Appl. Math., 2(3):237272, 2006.

[Pou08] Maurice Pouzet. When is the orbit algebra of a group an integral domain? Proof of a conjecture of P. J. Cameron. Theor. Inform. Appl., 42(1):83-103, 2008.

[PS01] Maurice Pouzet and Mohamed Sobrani. Sandwiches of ages. In Proceedings of the XIth Latin American Symposium on Mathematical Logic (Mérida, 1998), volume 108, pages 295-326, 2001.

[PT05] Maurice Pouzet and Nicolas M. Thiéry. Some relational structures with polynomial growth and their associated algebras. In Proceedings of FPSAC'05 Taormina, 2005. arXiv:0601256 [math.CO].

[PT08] Maurice Pouzet and Nicolas M. Thiéry. Some relational structures with polynomial growth and their associated algebras. In Youssef Boudabbous and Nejib Zaguia, editors, ROGICS'08: International Conference on Relations, Orders and Graphs: Interaction with Computer Science, 12-17 may, 2008 Mahdia, Tunisia. Nouha, 2008. arXiv:0801.4404v1 [math.CO].

[PT12] Maurice Pouzet and Nicolas M. Thiéry. Some relational structures with polynomial growth and their associated algebras II: Finite generation. 2012. In preparation, 20 pages.

[RN59] C. Ryll-Nardzewski. On the categoricity in power $\leqslant \aleph_{0}$. Bull. Acad. Polon. Sci. Sér. Sci. Math. Astr. Phys., 7:545-548., 1959. 
[Sch90] James H. Schmerl. Coinductive $\aleph_{0}$-categorical theories. J. Symbolic Logic, 55(3):1130-1137, 1990.

[SF96] R. Sedgewick and P. Flajolet. Analysis of algorithms. Addison Wesley, 42:192, 1996.

[Sta97] Richard P. Stanley. Enumerative combinatorics. Vol. 1, volume 49 of Cambridge Studies in Advanced Mathematics. Cambridge University Press, Cambridge, 1997. With a foreword by Gian-Carlo Rota, Corrected reprint of the 1986 original.

[TT04] Nicolas M. Thiéry and Stéphan Thomassé. Convex cones and SAGBI bases of permutation invariants. In Invariant theory in all characteristics, volume 35 of CRM Proc. Lecture Notes, pages 259-263. Amer. Math. Soc., Providence, RI, 2004. arXiv:0607380 [math.AC].

[Vat11] V. Vatter. Small permutation classes. Proceedings of the London Mathematical Society, 103(5):879-921, 2011. arXiv:0712.4006.

[vLW92] J. H. van Lint and R. M. Wilson. A course in combinatorics. Cambridge University Press, Cambridge, 1992. 